\title{
Physics opportunities with the Advanced Gamma Tracking Array: AGATA
}

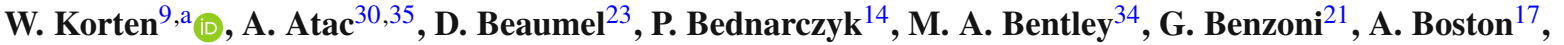 \\ A. Bracco ${ }^{20,21}$, J. Cederkäll ${ }^{18}$, B. Cederwall ${ }^{30}$, M. Ciemała ${ }^{14}$, E. Clément ${ }^{1}$, F. C. L. Crespi ${ }^{20,21}$, D. Curien ${ }^{31}$, \\ G. de Angelis ${ }^{15}$, F. Didierjean ${ }^{31}$, D. T. Doherty ${ }^{10}$, Zs. Dombradi ${ }^{6}$, G. Duchêne ${ }^{31}$, J. Dudek ${ }^{31}$, \\ B. Fernandez-Dominguez ${ }^{27}$, B. Fornal ${ }^{14}$, A. Gadea ${ }^{33}$, L. P. Gaffney ${ }^{17}$, J. Gerl ${ }^{4}$, K. Gladnishki ${ }^{28}$, A. Goasduff ${ }^{25}$, \\ M. Górska ${ }^{4}$, P. T. Greenlees ${ }^{12}$, H. Hess ${ }^{13}$, D. G. Jenkins ${ }^{34}$, P. R. John ${ }^{5}$, A. Jungclaus ${ }^{19}$, M. Kmiecik ${ }^{14}$, A. Korichi ${ }^{22}$, \\ M. Labiche ${ }^{3}$, S. Leoni ${ }^{20,21}$, J. Ljungvall ${ }^{22}$, A. Lopez-Martens ${ }^{22}$, A. Maj ${ }^{14}$, D. Mengoni ${ }^{24,25}$, B. Million ${ }^{21}$, \\ A. Nannini ${ }^{8}$, D. Napoli ${ }^{15}$, P. J. Nolan ${ }^{17}$, J. Nyberg ${ }^{32}$, A. Obertelli ${ }^{5}$, J. Pakarinen ${ }^{11,12}$, N. Pietralla ${ }^{5}$, Zs. Podolyák ${ }^{10}$, \\ B. Quintana ${ }^{26}$, R. Raabe ${ }^{16}$, G. Rainovski ${ }^{28}$, F. Recchia ${ }^{24,25}$, P. Reiter ${ }^{13}$, D. Rudolph ${ }^{18}$, J. Simpson ${ }^{3}$, Ch. Theisen ${ }^{9}$, \\ D. Tonev ${ }^{29}$, A. Tumino ${ }^{2,7}$, J. J. Valiente-Dobón ${ }^{15}$, O. Wieland ${ }^{21}$, K. Wimmer ${ }^{19}$, M. Zielińska ${ }^{9}$, \\ the AGATA Collaboration
}

${ }^{1}$ GANIL, CEA/DRF-CNRS/IN2P3, Bd. Henri Becquerel, BP 55027, 14076 Caen Cedex 05, France

${ }^{2}$ Laboratori Nazionali del Sud, INFN, Via S. Sofia 62, 95123 Catania, Italy

${ }^{3}$ Daresbury Laboratory, UKRI-STFC, Daresbury, Warrington WA4 4AD, UK

${ }^{4}$ GSI Helmholtzzentrum für Schwerionenforschung GmbH, Planckstrasse 1, 64291 Darmstadt, Germany

${ }^{5}$ Institut für Kernphysik, Technische Universität Darmstadt, Schlossgartenstrasse 9, 64289 Darmstadt, Germany

${ }^{6}$ Institute for Nuclear Research, Hungarian Academy of Sciences, Bem Square 18/c, 4026 Debrecen, Hungary

${ }^{7}$ Facoltá di Ingegneria e Architettura, Universitá di Enna Kore, 94100 Enna, Italy

${ }^{8}$ INFN, Sezione di Firenze, 50125 Florence, Italy

${ }^{9}$ Irfu, CEA, Université Paris-Saclay, 91191 Gif-sur-Yvette, France

${ }^{10}$ Department of Physics, University of Surrey, Guildford GU2 7XH, UK

${ }^{11}$ Helsinki Institute of Physics, 00014 Helsinki, Finland

12 Department of Physics, University of Jyvaskyla, P.O. Box 35, 40014 Jyvaskyla, Finland

${ }^{13}$ Institut für Kernphysik, Universität zu Köln, Zülpicher Straße 77, 50937 Cologne, Germany

${ }^{14}$ Institute of Nuclear Physics, Polish Academy of Sciences, 31-342 Kraków, Poland

${ }^{15}$ Laboratori Nazionali di Legnaro, INFN, Viale dell'Università, 2, 35020 Legnaro, Italy

${ }^{16}$ Instituut voor Kern- en Stralingsfysica, KU Leuven, 3001 Leuven, Belgium

17 Oliver Lodge Laboratory, University of Liverpool, Liverpool L69 7ZE, UK

18 Department of Physics, Lund University, 22100 Lund, Sweden

${ }^{19}$ Instituto de Estructura de la Materia, CSIC, Madrid, 28006 Madrid, Spain

${ }^{20}$ Dipartimento di Fisica dell'Università degli Studi di Milano, 20133 Milan, Italy

${ }^{21}$ INFN, Sezione di Milano, Via Celoria 16, 20133 Milan, Italy

${ }^{22}$ CSNSM, CNRS/IN2P3, Université Paris-Sud, Université Paris-Saclay, 91405 Orsay, France

${ }^{23}$ Institut de Physique Nucléaire, CNRS-IN2P3, Université Paris-Sud, Université Paris-Saclay, 91406 Orsay, France

${ }^{24}$ INFN, Sezione di Padova, 35131 Padua, Italy

25 Dipartimento di Fisica e Astronomia dell’Università degli Studi di Padova, 35131 Padua, Italy

${ }^{26}$ Departamento de Física Fundamental, Universidad de Salamanca, 37008 Salamanca, Spain

${ }^{27}$ Universidade de Santiago de Compostela, 15754 Santiago de Compostela, Spain

28 Department of Atomic Physics, Faculty of Physics, University of Sofia, 1164 Sofia, Bulgaria

${ }^{29}$ Institute for Nuclear Research and Nuclear Energy, Bulgarian Academy of Sciences, 1784 Sofia, Bulgaria

${ }^{30}$ Department of Physics, KTH Royal Institute of Technology, 10691 Stockholm, Sweden

${ }^{31}$ Université de Strasbourg, CNRS, IPHC UMR 7178, 67037 Strasbourg, France

${ }^{32}$ Department of Physics and Astronomy, Uppsala University, 75120 Uppsala, Sweden

33 Instituto de Física Corpuscular IFIC, CSIC-University of Valencia, 46980 Paterna, Valencia, Spain

${ }^{34}$ Department of Physics, University of York, Heslington, York YO10 5DD, UK

35 Department of Physics, Ankara University, 06100 Tandogan, Ankara, Turkey

\footnotetext{
a e-mail: w.korten@cea.fr (corresponding author)
} 
Abstract New physics opportunities are opening up by the Advanced Gamma Tracking Array, AGATA, as it evolves to the full $4 \pi$ instrument. AGATA is a high-resolution $\gamma$-ray spectrometer, solely built from highly segmented high-purity Ge detectors, capable of measuring $\gamma$ rays from a few tens of $\mathrm{keV}$ to beyond $10 \mathrm{MeV}$, with unprecedented efficiency, excellent position resolution for individual $\gamma$-ray interactions, and very high count-rate capability. As a travelling detector AGATA will be employed at all major current and near-future European research facilities delivering stable and radioactive ion beams.

\section{Introduction}

Nuclear structure studies far from stability are entering into a high-precision era with increased intensities and purity of radioactive ion beams and new methods to produce exotic nuclei using stable beams. High-resolution $\gamma$-ray spectroscopy is the only method capable of unravelling the complex nature of excited states and has therefore always played a prominent role in the understanding of nuclear structure. Improved efficiency and sensitivity of the instruments are mandatory to focus on essential observables to validate the theoretical predictions and guide future developments, This has led to a continuous improvement of the instrumentation, from the High-Purity Germanium (HPGe) multi-detector arrays of the 1990s (e.g., [1,2] in Europe, Gammasphere [3] in the USA), through the first arrays consisting of segmented HPGe detectors (e.g., MINIBALL [4,5], EXOGAM [6] in Europe, Gammasphere in the USA), to the development of the Advanced Gamma Tracking Array (AGATA) [7], a $4 \pi$ spectrometer solely built from position-sensitive HPGe detectors. Parallel developments are also being pursued in the USA leading to the realisation of the Gamma-Ray Energy Tracking Array (GRETA) [8,9].

AGATA is a major European project to develop, build, and operate a world-leading precision $\gamma$-ray detection instrument for in-beam studies of nuclei. AGATA uses a technique known as $\gamma$-ray tracking, which relies on determining every $\gamma$-ray interaction point in any of the HPGe detectors so that the whole path of a $\gamma$ ray can be tracked and used to measure not just the energy, but also the angle at which the original $\gamma$ ray was emitted. The technique relies on segmentation of Ge-detector contacts and digital signal processing to perform pulse-shape analysis enabling the interaction positions to be determined with high precision. The path of the $\gamma$ ray is then reconstructed from the Compton-scattering formula, allowing each individual $\gamma$ ray (out of many incident on the detector) to be tracked and recorded. A recent review of the technical advances in $\gamma$-ray tracking can be found in [10]. Gamma-ray spectroscopy benefits hugely from tracking since the Compton suppression shields, which surround

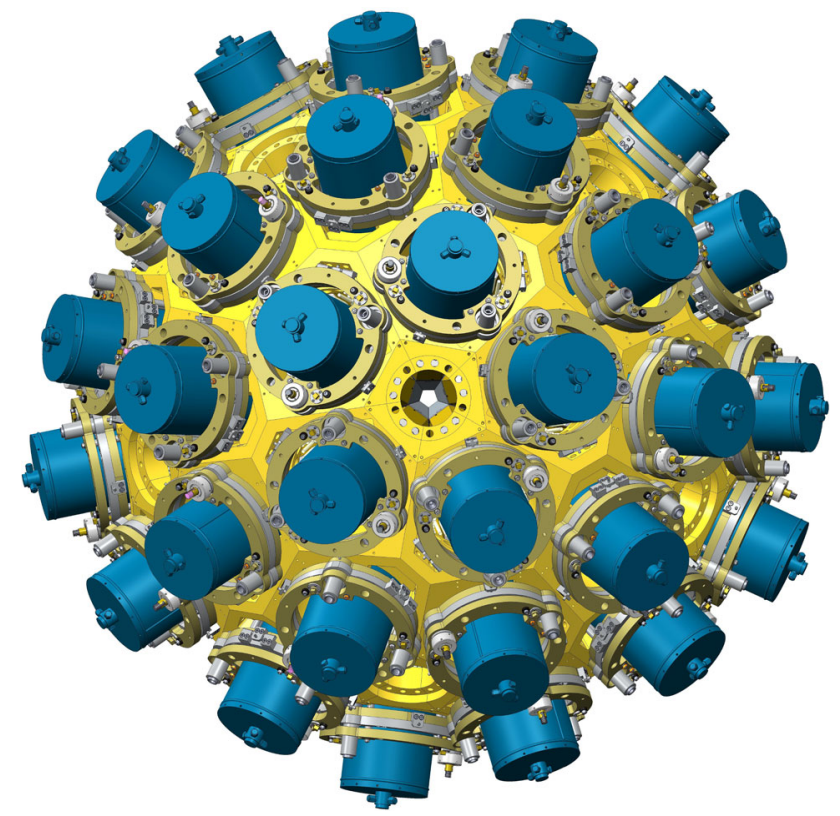

Fig. 1 Artist's view of the $4 \pi$ AGATA spectrometer showing the mechanical holding frame (yellow) and cryostat dewars (blue) of the Ge detectors

each individual Ge crystal and are used to improve the Ge spectrum quality, e.g., [1-3], are no longer required. The entire $4 \pi$ solid angle can thus be filled with closely-packed Ge detectors, vastly increasing the efficiency of the array, even for a very high multiplicity of (simultaneously emitted) $\gamma$-rays. Tracking also, crucially, allows precise Doppler correction of the measured $\gamma$-ray energies from fast moving nuclei.

In its complete $4 \pi$ geometry AGATA will be composed of 18036-fold segmented, hexagonal-shaped and tapered HPGe crystals, each one situated at $23.5 \mathrm{~cm}$ from the source of the photons of interest (see Fig. 1).

AGATA is a truly universal high-resolution spectrometer, capable of measuring $\gamma$ rays from a few tens of $\mathrm{keV}$ to beyond $10 \mathrm{MeV}$, with unprecedented efficiency, excellent position resolution for individual $\gamma$-ray interactions and correspondingly unparalleled angular resolution, and very high count-rate capability. These features will give rise to a resolving power that is in specific cases up to two orders of magnitude larger than current arrays, and allow AGATA to be operated in diverse environments such as using relativistic beams from the FAIR/Super-FRS facility $[11,12]$, high-intensity ISOL beams from the secondgeneration Radioactive Ion Beam (RIB) facilities (HIEISOLDE [13], SPES [14], SPIRAL2 [15]), and at the highintensity stable beam facilities at GANIL [15], JYFL [16], and LNL [17].

AGATA started as an R\&D project in the late 1990s and the AGATA demonstrator became operational in 2009. Steadily 


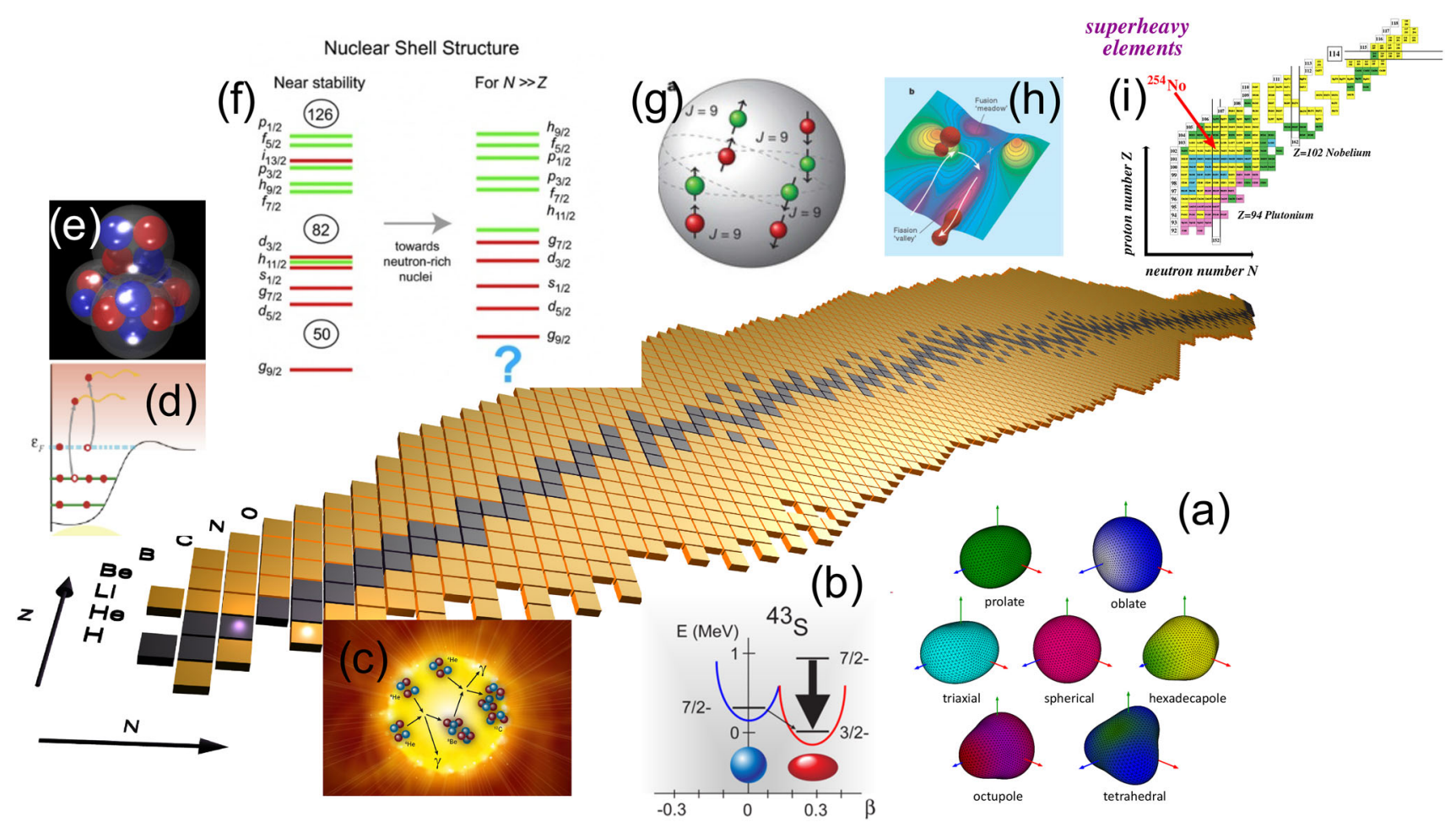

Fig. 2 Artist's view of the nuclear landscape illustrating some of the key properties that can be studied using high-resolution $\gamma$-ray spectroscopy: a the variety of nuclear shapes, $\mathbf{b}$ shape coexistence and isomerism, $\mathbf{c}$ reactions of astrophysical interest, $\mathbf{d}$ coupling to the contin-

growing over the years, AGATA has been in constant use for experiments. Currently a solid-angle coverage of approximately $1 \pi$ has been achieved. Exciting new results were obtained in campaigns at LNL (2009-2011), GSI (20122014) and GANIL (since 2015). This success led to the recommendation in the 2017 NuPECC Long-Range Plan [18] that "the timely completion of the full AGATA spectrometer and the provision of adequate support and maintenance are of the highest importance to address the exciting science programme at both the stable and radioactive beam facilities".

This paper describes new physics opportunities opened up by AGATA. In Sect. 2 the various physics cases are described for which the use of AGATA will be pivotal. Some examples are indicated in Fig. 2 and include:

- studying the evolution of the nuclear shell structure and the occurrence of new magic numbers,

- understanding the microscopic origin of nuclear deformation and the interplay between single-particle and collective degrees of freedom,

- searching for exotic and extreme shapes (e.g., hyperdeformation), uum of unbound states, $\mathbf{e}$ cluster structure in nuclei, $\mathbf{f}$ evolution of the shell structure, $\mathbf{g}$ nuclear superconductivity, $\mathbf{h}$ understanding nuclear fusion and fission reactions, and $\mathbf{i}$ the journey towards the heaviest elements

- establishing shape coexistence and shape transitions, and understanding the mechanisms leading to their appearance,

- testing theoretical predictions for neutron and proton skins,

- probing the nature of pair correlations and investigating how angular momentum is generated,

- measuring the degree of isospin-symmetry breaking,

- finding fingerprints of chaotic behaviour in nuclei,

$-\ldots$

In Sect. 3 more specific physics cases and simulations of experimental investigations are described that will profit from the unique capabilities of AGATA combined with specific advantages of the anticipated AGATA host laboratories.

\section{Physics cases}

2.1 The nuclear shell structure and its evolution

In the vicinity of the valley of stability "magic" numbers are well established for nuclides with $Z=2,8,20,28,50,82$, and $N=2,8,20,28,50,82$ and 126 . For these nucleon 

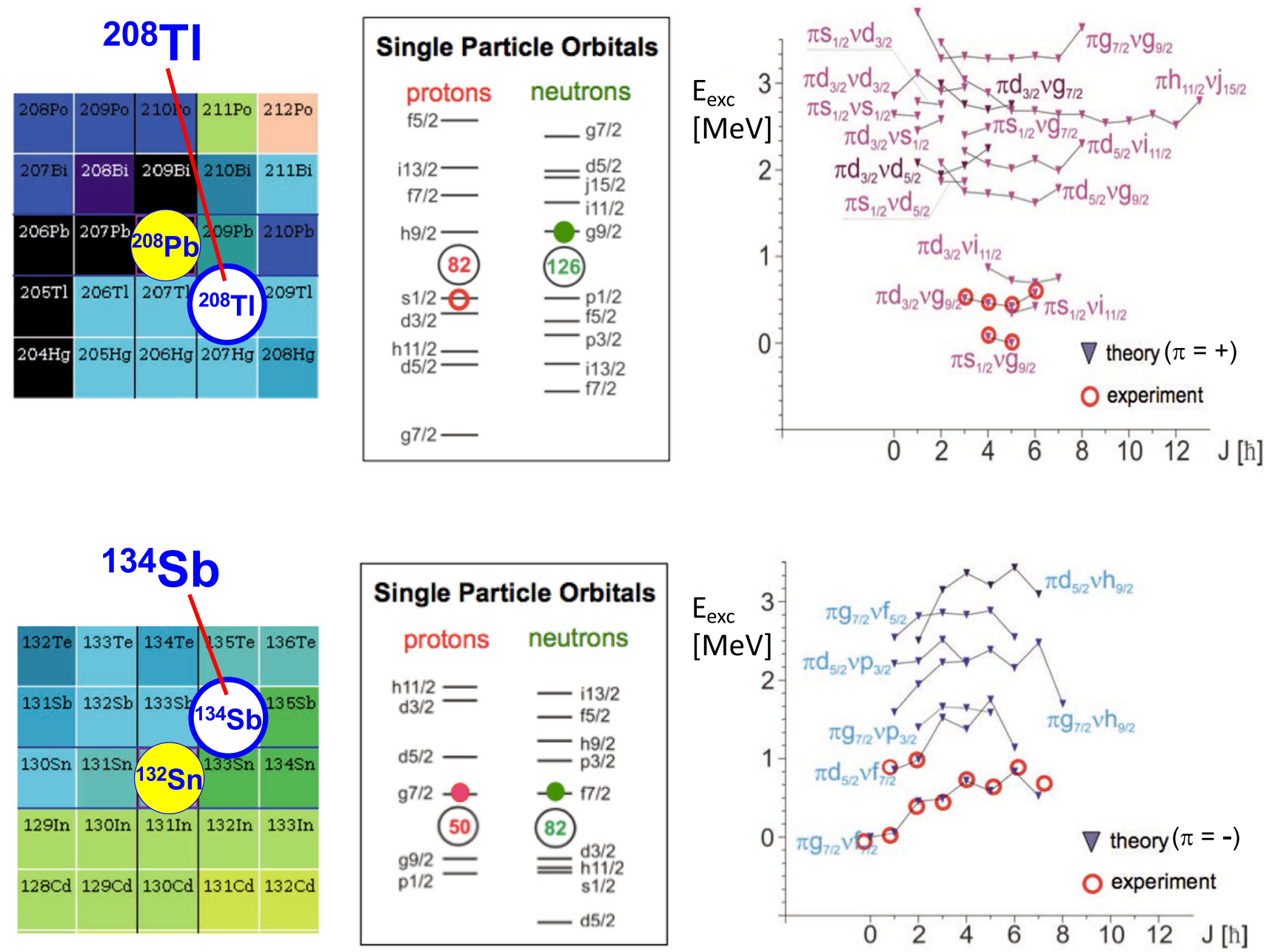

Fig. 3 Direct information on the proton-neutron interaction can be obtained, for example, from odd-odd nuclei around double shell closures, by studying multiplets arising from correlations between valence particles. Of special interest are nuclei like (i) ${ }^{134} \mathrm{Sb}$, located north-east of ${ }^{132} \mathrm{Sn}$, with one proton valence particle and one neutron valence particle, and (ii) the one proton hole-one neutron particle nucleus ${ }^{208} \mathrm{Tl}$, situated south-east of ${ }^{208} \mathrm{~Pb}$. Predictions for the multiplets up to $3.5 \mathrm{MeV}$ excitation energy in ${ }^{134} \mathrm{Sb}$ (negative parity) and ${ }^{208} \mathrm{Tl}$ (positive parity), obtained from shell-model calculations using realistic interactions [19], are shown on the right hand side. In both cases the experimental information is rather scarce. Only members of the lowest-lying multiplets are known. Higher-lying states belonging to other multiplets and being characterised by more significant configuration mixing can be established with AGATA, which will allow testing their diagonal and offdiagonal matrix elements and those of the proton-neutron interaction in much greater detail. All this information will become accessible with AGATA in hitherto unknown territories

dence of the in-medium nucleon-nucleon interaction. There are two main mechanisms predicted to drive shell evolution in nuclei: monopole migration and shell quenching due to a softening of the potential shape by excessive neutrons. There is firm evidence in light and medium-heavy nuclei that the neutron shells erode: For example the $N=8,20,28$ shell gaps give way to new magic numbers at $N=14,32,34, .$. , and the expected semi-magic nuclei, e.g., ${ }^{32} \mathrm{Mg},{ }^{42} \mathrm{Si}$, become deformed and lead to so-called islands of inversion. It will be very important to explore this evolution in heavier nuclei, namely to search for such islands below ${ }^{78} \mathrm{Ni}$ and ${ }^{132} \mathrm{Sn}$, where the spin-orbit interaction is more pronounced. 
The size of a shell gap and the identification of the corresponding mechanism responsible for its change can be inferred in some cases from the observation of lowlying core-breaking states, or deformed $0^{+}$states in eveneven nuclei, resulting from a delicate balance between particle excitation and correlation energy. New isomers, longer-living excited states typically in the range of ns$\mu \mathrm{s}$, which are often challenging to identify experimentally, can also be associated with the phenomenon of shell evolution.

In contrast to stable and near stable nuclei, where a strong proton-neutron interaction is a key ingredient in inducing deformation of the whole nucleus, decoupling between the valence neutrons and the proton core may occur in very neutron-rich nuclei. Decoupling or strong polarisation effects in nuclei can be searched for by measuring the evolution of the electric quadrupole transition strength, $B(E 2)$, along singly-magic isotopic chains.

Experiments with AGATA will lead to breakthrough studies related to the above properties by combining the specific strength of different facilities with the most advantageous reaction mechanisms and observables: knock-out and relativistic Coulomb excitation at FAIR, Coulomb excitation and transfer reactions at the ISOL facilities, as well as spectroscopic studies using fusion, fission or deep-inelastic reactions at stable beam facilities. For nuclei, which possess or lack only one nucleon in otherwise empty or filled shells, the energies of single-particle and single-hole states can be extracted, which are important empirical parameters in any microscopic description of nuclear properties of atomic nuclei. Nuclei with two particles outside a doubly-magic core bring the most direct information on the correlations between pairs of nucleons occupying orbitals close to the Fermi surface. Even-even nuclei provide the effective interaction between like nucleons, while the odd-odd isotopes give access to the proton-neutron $(\pi \nu)$ two-body matrix elements.

The interplay between single-particle excitations and collective responses of the nucleus (phonons in particular) generates a multifaceted scenario of nuclear excitations, which can be studied in their simplest form in systems composed of one valence particle and a doubly-magic core. These types of couplings between nucleons and phonons are major sources of partial occupancies of nucleonic orbitals and constitute doorways to more complex excitations, all the way up to damping phenomena in Giant Resonances. Among a number of very interesting cases, the spectroscopy of one/twovalence-particle/hole systems with respect to the doublymagic ${ }^{132} \mathrm{Sn}$ core, is probably the most accessible for European ISOL facilities, where intense and pure ${ }^{132}$ Sn-like beams will be available.

The coupling of the AGATA array with a recoil spectrometer and light charged-particle detection devices will allow very selective studies of neutron-rich systems populated by transfer reactions. Using the excellent position resolution of AGATA, angular distribution and polarisation measurements will allow firm spin and parity assignment of the excited states. An assessment of their single-particle and collective properties using a combination of lifetime measurements, as well as static magnetic and quadrupole moments will lead to a complete characterisation of their properties and open a new high-precision area in nuclear structure studies of nuclei far from the valley of stability.

\subsection{Exotic nuclei and clusterisation phenomena}

Weakly-bound systems provide a sensitive test of the nuclear force, and the regions in the vicinity of the drip-lines offer unique opportunities to extend our understanding of this interaction. Light nuclei are a test bench for the most advanced theoretical approaches and play a key role in nuclear astrophysics. Recent developments in ab-initio many-body methods allow their application to light exotic to best constrain nuclear forces. In turn, detailed experimental observations will shed light on the question as to which higher-order terms of the nuclear interaction (threebody forces, etc.) are essential for a correct description of nuclear properties.

On the neutron-deficient side of the valley of stability, the instability against spontaneous proton emission is masked by the Coulomb barrier, allowing these nuclei to have "measurable" lifetimes (ns to $\mu \mathrm{s}$ ) and their excited states to decay by $\gamma$-ray emission. Therefore, these nuclei are the ideal laboratory to study "particle-unbound" states using $\gamma$ rays, but also the competition between charged-particle and $\gamma$-ray emission.

On the neutron-rich side, phenomena of clusterisation are emerging; near threshold states are of paramount importance in astrophysics (such as the Hoyle state in stable ${ }^{12} \mathrm{C}$ ) and continuum states have a large impact on the structure of the nucleus. From the theoretical point of view, these necessitate the merging of structure and reaction descriptions.

Electromagnetic transitions are a very sensitive probe of the wave functions. Studying bound states of exotic nuclei, for instance ${ }^{20} \mathrm{O}$, will provide precision tests of the nuclear interaction. AGATA will also allow studies of electromagnetic decays from unbound states or resonances with typical decay branches as low as $10^{-3}-10^{-5}$. The latter constitute an almost unexplored territory, ideal for investigations with AGATA coupled to light charged-particle detectors, using intense stable beams. For example, reactions such as ${ }^{6,7} \mathrm{Li}$ on ${ }^{6,7} \mathrm{Li},{ }^{9} \mathrm{Be},{ }^{10,11} \mathrm{~B}$, and ${ }^{12,13} \mathrm{C}$ targets, will lead, after a single proton evaporation, to the population of unbound states in ${ }^{13} \mathrm{~B},{ }^{15} \mathrm{C},{ }^{17,19} \mathrm{O}$, and ${ }^{16,17} \mathrm{~N}$, respectively. 


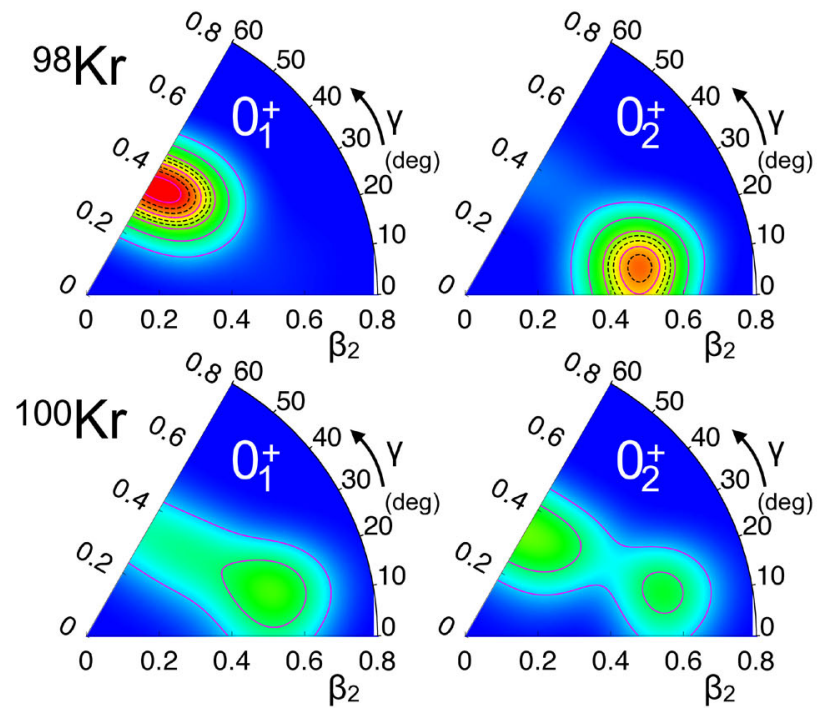

Fig. 4 Shape coexistence occurs when different microscopic configurations correspond to similar binding energies, as illustrated by the wave functions of $0^{+}$states in the neutron-rich krypton isotopes beyond $N=60$ (left), where oblate and prolate shapes are expected to coexist. Low-energy Coulomb excitation is an ideal tool to pin down their structure. These different configurations may also appear as long-lived

\subsection{Shape coexistence}

The shape of an atomic nucleus is governed by the interplay of macroscopic, liquid-drop-like properties of the nuclear matter and microscopic shell effects. While closed-shell nuclei always exhibit spherical shapes in their ground state, nucleons residing in open shells tend to deform the mass distribution, with quadrupole deformation being the dominant degree of freedom. The shape of a nucleus depends on the details of the microscopic wave function of the state considered. Thus it may change, not only as a function of the number of nucleons, but it may also vary from one state to another within the same nucleus; the latter is known as shape coexistence. Establishing shape coexistence is challenging and requires highly-refined experimental techniques. Nevertheless, it provides one of the most demanding and stringent tests of modern nuclear theories and models. It is for this reason that studies of this phenomenon are at the forefront of nuclear structure research.

Typically, shape coexistence arises when the nuclear potential energy surface in the deformation space exhibits minima associated with different shapes. When the energy barrier separating such minima is sufficiently high, it may lead to the appearance of shape isomers, i.e., long-lived states with their electromagnetic decay hindered by the necessary shape change, like the well-known fission isomers in the actinides. Striking manifestations of shape coexistence can also be found in nuclei on the shores of the islands

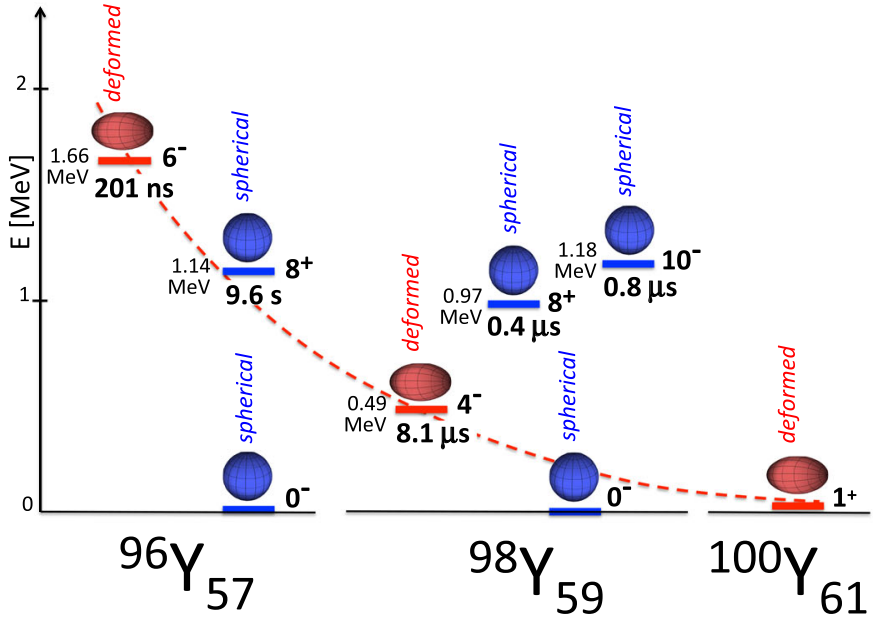

isomers, as observed in nearby $\mathrm{Y}$ isotopes (right). To reach such states, cluster-transfer reactions can be employed. Besides its high efficiency, the unprecedented position resolution of AGATA is needed to counteract the Doppler broadening. Its high-rate capability will ensure high throughput despite possible occurrence of intense contaminations in RIB experiments

of inversion, and in open-shell nuclei where rapid shape changes occur. Very neutron-rich nuclei exhibit a neutron skin, which may assume a different shape and may deform more easily than the core. This leads to an enhancement of the isovector components in electromagnetic decays. Correspondingly, unusual shape-coexistence phenomena may be observed, whereby the isovector shapes will co-exist with the isoscalar ones. Such effects are abundantly predicted for small-amplitude vibrational states. However, the same arguments also apply for all kinds of low-lying collective excitations, which should probably be described by novel types of collective models that take the isospin degrees-of-freedom explicitly into account.

Experiments with AGATA will create a multitude of opportunities to study the evolution of nuclear shapes and shape coexistence. Coulomb-excitation studies with stable and radioactive beams will allow identification of different shapes by measuring quadrupole moments of shortlived excited states and determining deformation parameters, in particular of $0^{+}$states, from quadrupole shape invariants. Direct determination of very weak, retarded $\gamma$ decay branches between different configurations will provide insight into the microscopic origin of nuclear deformation, which can be analysed on the basis of, for example, the largescale shell-model calculations. Complementary information will be obtained by following the evolution of the nuclear shape along isotopic chains. One of the key regions for this type of investigation is that of the neutron-rich nuclei from 
Kr to $\mathrm{Zr}$ with $Z=36-40$, near $N=60$, (see Fig. 4), where the most dramatic shape change in the nuclear chart has been observed, with typical characteristics of a quantum-phase transition. This phenomenon has also a strong impact on the $r$-process nucleosynthesis path.

\subsection{Higher-order nuclear deformation}

Octupole collectivity is the most prominent exotic deformation in nuclei and most pronounced just above the magic numbers of protons and neutrons. In doubly-magic nuclei such as ${ }^{40} \mathrm{Ca},{ }^{132} \mathrm{Sn}$, and ${ }^{208} \mathrm{~Pb}$, the first excited $3^{-}$state is at a comparable, if not lower, energy as the first excited $2^{+}$ state. Quadrupole vibrations are invoked to explain the first $2^{+}$state in these nuclei and octupole vibrations, by analogy, are responsible for the first $3^{-}$state. Since there is no coupling between these two vibrational modes at low energy, a very weak $E 1$ transition between them is observed. In quadrupoleoctupole deformed systems, a strong coupling between the two degrees of freedom gives rise to enhanced $E 1$ transitions. Large electric octupole $B(E 3)$ values of the order of 30-80 W.u. are, for example, observed in ${ }^{224,226}$ Ra. Studying the transitional region between these two modes, i.e., octupole-vibrational nuclei at and near doubly-magic nuclei and octupole-deformed nuclei to the north-east of these, will shed light on the evolution of octupole collectivity. Of particular importance are the energies of negative-parity states in transitional nuclei and the $\mathrm{B}\left(E 3 ; 0_{1}^{+} \rightarrow 3_{1}^{-}\right)$transition strengths, proportional to the square of the octupole moment, $Q_{3}$.

Octupole-deformed nuclei have implications beyond nuclear structure physics, e.g., for experiments aiming at measuring an atomic non-zero Electric Dipole Moment (EDM), which use octupole-deformed nuclei as a probe. In odd-mass systems with a significant octupole moment, $Q_{3}$, an increase in the Schiff moment of up to three orders of magnitude is expected compared with the current best limit in ${ }^{199} \mathrm{Hg}$. It is this nuclear Schiff moment that gives rise to the EDM in the atomic system. Any observation of a non-zero atomic EDM would require new physics beyond the standard model. Nuclear physics input to the calculation and interpretation of the Schiff moment needs to come from theory and experiment, with the energy difference between paritydoublets in odd-mass nuclei currently being the biggest unknown. Direct measurements of $Q_{3}$ in odd-mass nuclei are very desirable, though a simpler measurement of this quantity in the neighbouring even-mass isotopes can already guide the theoretical calculations.

The research on new exotic nuclear symmetries includes the search for tetrahedral and octahedral symmetries and the underlying nuclear deformation (see Fig. 5). Some of the intriguing new phenomena predicted to accompany tetrahedral symmetry are four-fold degeneracies of the nucle-
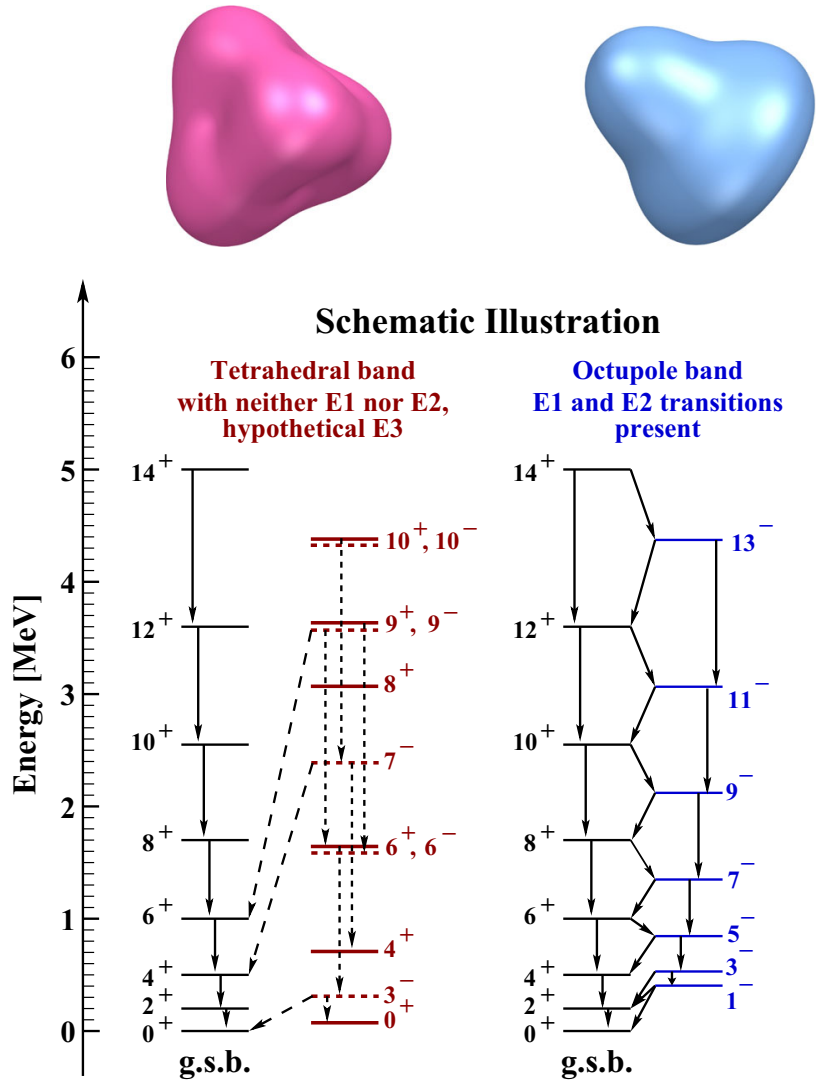

Fig. 5 Schematic illustration comparing the characteristic level schemes of an octupole-deformed nucleus (right) with staggered levels of opposite parity and decaying via enhanced $E 1$ and $E 2$ transitions and a tetrahedral nucleus (left) with characteristic parity doublets (at spins $6,9,10)$ and decaying predominantly through E3 transitions. While rigid octupole shapes have been observed in certain mass regions, the existence of tetrahedral nuclei still needs to be established. AGATA is the ideal tool to look for these unusual decay patterns

onic orbitals, rather than the usual two-fold spin-up/spindown Kramers degeneracy, or 16-fold degeneracies of the particle-hole exited states and 32-fold degeneracies of the two-particle two-hole excited states. New classes of isomeric configurations could also be expected, producing possibly new waiting-point nuclei, important for the astrophysical modelling of the stellar processes.

The unusual electromagnetic signals from those very special configurations are expected to be very weak and specific analysis techniques have to be introduced, where the application of AGATA will be particularly relevant. In the exact tetrahedral and octahedral symmetry limit, nuclei would exhibit neither $E 2$ nor $E 1$ transitions. However, exact symmetries are never really present in nuclei due to different symmetrybreaking processes, such as zero-point motion around the corresponding minimum in the quadrupole deformation surface as well as Coriolis coupling of the high-j orbitals. Consequently, AGATA will be essential in detecting the weak $E 2$ and $E 1$ signals originating from the configurations with par- 
tially broken symmetries. Similarly, the enhanced E3 transitions between specific states along the tetrahedral bands can be searched for. Such structures are predicted in stable nuclei, for example ${ }^{96} \mathrm{Zr}$ and ${ }^{152} \mathrm{Sm}$, as well as in exotic isotopes $\left({ }^{104} \mathrm{Zr}\right)$.

\subsection{Isospin symmetry}

Isospin symmetry is based on the facts that protons and neutrons are (almost) identical particles, and that nuclear forces are nearly charge independent. Although it is known that isospin symmetry is broken to a small extent by the strong interaction, in a more significant way by the weak interaction and, most significantly, by the electromagnetic interaction, the isospin formalism, characterising nuclear states by the isotopic spin ("isospin") quantum number $\mathrm{T}$, remains an extremely powerful tool to understand the structure of nuclei.

The concept of isospin symmetry is most clearly established in so-called "mirror nuclei", i.e., nuclei of the same mass, for which the number of protons and neutrons is exchanged, resulting in their structure being very similar. More generally, isobaric nuclei (with the same mass number $A$ ) have corresponding levels, which can be grouped into multiplets of states of common isospin. However, the isospin symmetry-breaking terms of the nuclear Hamiltonian should lead to each nuclear state having, in addition to its main component of isospin $\mathrm{T}$, minor components of a different isospin. The amount of isospin mixing, as derived from experiment, can be understood as a measure of the magnitude of the symmetry violation. The breaking of the isospin symmetry by the Coulomb force increases with $Z$ and for a given mass it is at its maximum for $N=Z$ nuclei. The study of the heavier nuclei with $N \approx Z$ is thus of fundamental importance. With AGATA in its final configuration $N=Z$ nuclei up to the doubly-magic self-conjugate ${ }^{100} \mathrm{Sn}$ come into reach for detailed spectroscopic studies (see Fig. 6).

Isospin symmetry breaking can also be studied by examining the isospin mixing of nuclear states, which can be directly accessed by measuring the isospin-forbidden $E 1$ transitions (with $\Delta T=0$ ) in $N=Z$ nuclei, making use of the following rules:

- $E 1$ transitions between states of equal isospin are forbidden in $N=Z$ nuclei,

- $B(E 1)$ values between corresponding states in mirror nuclei should be equal,

- Mirror $\gamma$-ray transitions with $\Delta T=1$ and of any multipolarity should have equal reduced strength,

- The reduced matrix elements of E2 $\gamma$-ray transitions between analogue states of an isobaric multiplet should vary linearly with isospin projection $(N-Z)$, and with a slope given by the isovector effective charge.
In particular, the last rule has not been investigated to any extent in nuclei beyond the $f p$ shell. For the $E 1$ transitions, in addition to discrete spectroscopy studies, the giant dipole resonance (GDR), where the maximum $E 1$ strength is concentrated, is ideal for searching for small effects in the breaking of the associated selection rules. The dependence of the isospin mixing on temperature can, for example, be deduced from $N=Z$ compound nuclei produced at finite temperature, and then extrapolated to zero temperature using model predictions. This type of studies was successfully done for ${ }^{80} \mathrm{Zr}$ with AGATA in its earliest implementation.

Another interesting aspect of heavy mirror nuclei is the possibility to search for exotic matter distributions in the nucleus. The theoretical predictions for proton skins can be tested by means of Coulomb energy differences of isobaric analogue states, since the Coulomb repulsion between the protons in the nucleus is directly related to their spatial distributions. Level differences have been measured as a function of angular momentum in heavy mirror nuclei. When data for $T=1$ bands in $N=Z$ odd-odd nuclei and in the more exotic $T_{z}=-1$ members of the multiplets, e.g., those with $N=Z-2$, became available, it was realised that the perturbative inclusion of the Coulomb interaction among protons (with charge-invariant model wave functions) was not sufficient to explain the measured energy differences. While it was possible to add phenomenological terms to the shell-model interactions to describe the effect, further experiments with AGATA will be essential to clarify the origin of these terms.

\subsection{High-spin states, extreme deformation, and giant collective modes}

When a deformed nucleus rotates, the energy of the "intruder" states from higher-lying major shells is significantly affected by the rotational frequency. This gives rise to characteristic particle configurations on which many rotational bands are built. The study of rotational band properties may be used to establish these configurations and hence determine the shell energies in nuclei far from stability. Moreover, in the de-excitation of an intruder state along a rotational band by the emission of $\gamma$ rays, neutron-rich nuclei may at some point become unstable against neutron emission, leading to appearance of the novel phenomenon of a $\gamma$ delayed neutron emission. An analogous proton emission has already been observed in neutron-deficient nuclei. On the neutron-rich side, the particle emission is not hindered by the Coulomb barrier. Such measurements would immediately give information on the position of the intruder state within the sequence of single-particle levels. By studying high-spin states, $K$-isomers, and rotational bands in exotic nuclei it will also be possible to determine the properties and positions of single-particle levels and hence gain access to the shell properties of these nuclei. 

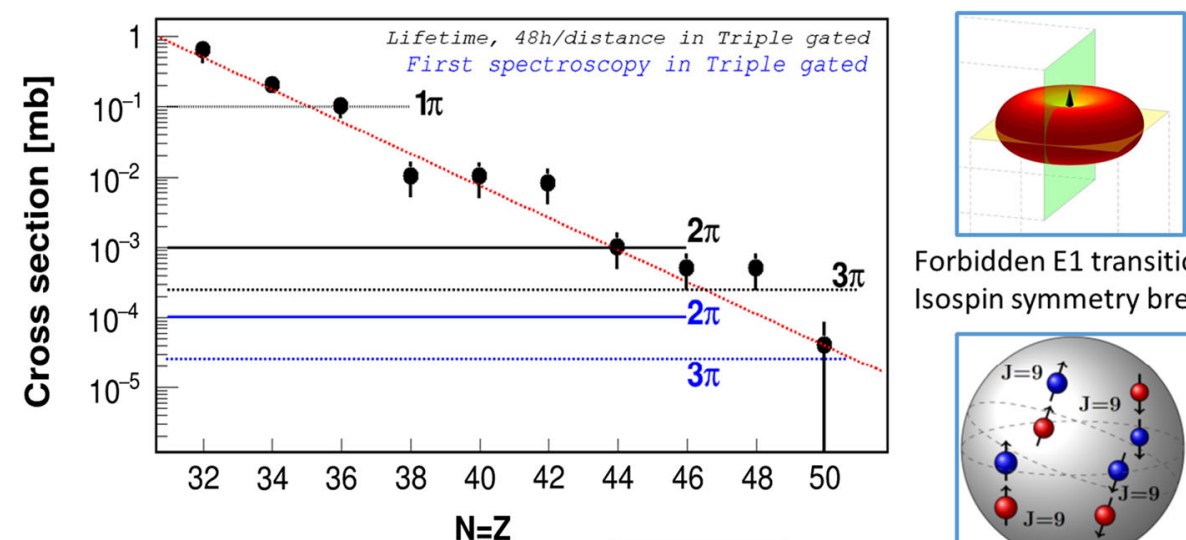

Forbidden E1 transition

Isospin symmetry breaking
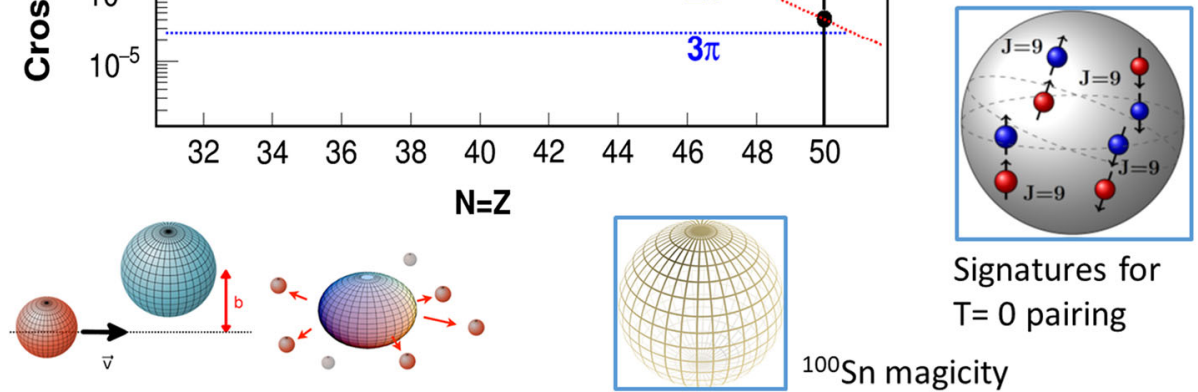

Signatures for

$\mathrm{T}=0$ pairing

${ }^{100} \mathrm{Sn}$ magicity

Fig. 6 The evolution of AGATA will allow more and more exotic $N=Z$ nuclei to be reached. AGATA coupled to a $1 \pi$ neutron detector and a charged-particle array will allow spectroscopy towards doublymagic ${ }^{100} \mathrm{Sn}$, to determine transition probabilities by lifetime measurement of excited states in ${ }^{96} \mathrm{Cd}$, and angular distribution and polarisation measurements of ${ }^{92} \mathrm{Pd}$. The figure illustrates the estimated limits for a plunger lifetime measurement (black, $48 \mathrm{~h}$ per distance) and first spectroscopy (blue, 14 days) as a function of the solid angle covered by AGATA. Beam intensities of $10 \mathrm{pnA}$ (for ${ }^{40} \mathrm{Ca},{ }^{50} \mathrm{Cr}$, and ${ }^{58} \mathrm{Ni}$ ) and a medium $\gamma$-ray multiplicity are considered. The cross sections are taken from [20] and experiments at GANIL

and higher efficiency at energies of $4 \mathrm{MeV}$ and above, where we expect another gain of an order of magnitude. All these studies require the availability of high-intensity stable ion beams to reach ultra high-spin states in exotic nuclei, or even intense neutron-rich RIBs, which are expected to populate states of even higher angular momentum due to the increase in the fission barrier with neutron number.

Non-axial nuclear shapes, and in particular triaxial configurations, may give rise to appearance of chirality effects. Chirality arises when a physical object cannot be superposed with its mirror image via continuous movement, as for example human left and right hands or a triplet of vectors. The role of chirality in nuclear structure can be studied in specific odd-odd nuclei, where the three vectors needed to pose the chirality problem are the two individual angular momenta of the odd proton, $j_{p}$, and the odd neutron, $j_{n}$, together with the collective-rotation vector $\mathrm{R}$, all three aligned with three different axes. In principle, such a triplet of distinct vectors in the nuclear microscopic mean-field theory generates no dynamical distinctions. It implies that a nucleus rotating 'from the left to the right' or 'from the right to the left' should present strictly identical energy relations. Experimental observation of non-identical rotational sequences is a sign of mechanisms going beyond the mean field and the scale of the symmetry breaking provides important input for more advanced modelling and ideas about spontaneous symmetry breaking. While the effects of chirality have been observed in the excitation energy spectra of certain odd-odd nuclei, 


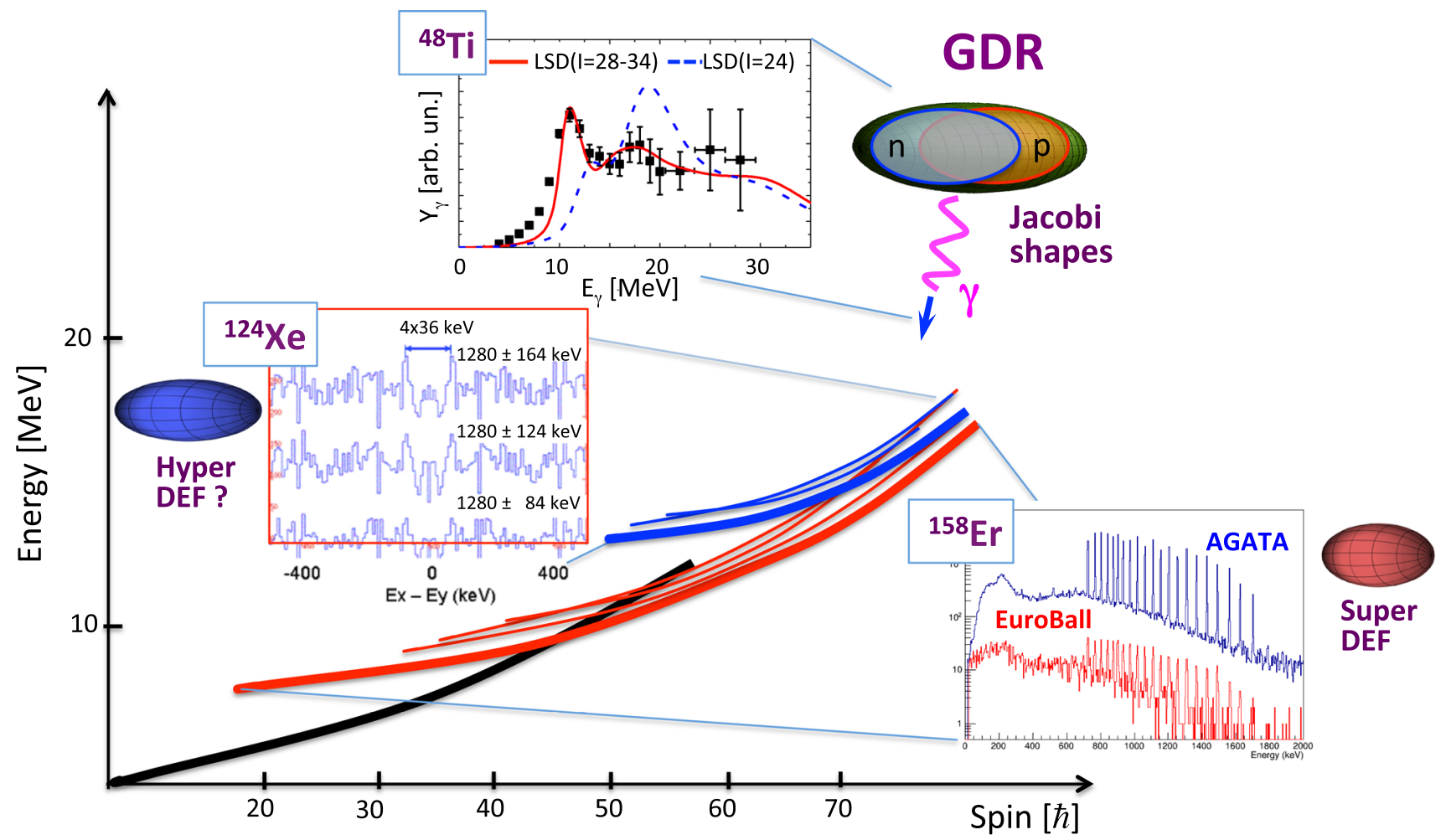

Fig. 7 Illustrations of the nuclear response at the highest spins and simulations of spectra for selected examples from the previous generation of gamma-ray spectrometers, such as EUROBALL, compared with $4 \pi$ AGATA: Population of highly elongated structures in cold nuclei (e.g., the lowest-energy triaxial strongly deformed band in ${ }^{158} \mathrm{Er}$ and the possibly hyper-deformed ridge structures in ${ }^{124} \mathrm{Xe}$ [21]), but also in

their spectroscopy is extremely complicated. Further detailed investigations are needed to fully understand the influence of chirality.

Compelling evidence for triaxiality in nuclei was established with the observation of wobbling excitations in rotational structures at high spin in $\mathrm{Lu}$ nuclei around mass $A \sim 160$. More recently, several triaxial strongly deformed bands were also observed in ${ }^{158} \mathrm{Er}$ and neighbouring nuclei. These bands extended the level structure of nuclei in this region beyond the classic band termination states at spin $50 \hbar$ to ultra-high spin $(\sim 70 \hbar)$. However, like many cases of superdeformation, a direct connection to the yrast states has not been established. As a result their spin values remain an estimate. It is interesting that some recent theoretical calculations predict these structures at much higher spin than previously expected. Several questions remain thus open in this high spin domain, including, what is the spin and parity of these bands? Are these ultra-high spin states a common feature in other rare-earth nuclei? Can we observe wobbling excitations in these structures? All these questions can only be answered using the full $4 \pi$ AGATA array. warm rotating nuclei via the decay of the low-energy component of the Giant Dipole Resonance (GDR) built on a hot rotating nucleus possessing a very elongated Jacobi shape (e.g., in ${ }^{48} \mathrm{Ti}$ [22]). AGATA in its $4 \pi$ configuration, making use of highest-fold $\gamma^{n}$ coincidences, will exceed significantly the performance of EUROBALL and will therefore enable in-depth studies of these processes

\subsection{Pygmy resonance excitations}

The presence of dipole strength at low excitation energy has been often associated with the possible existence of a new collective mode: the Pygmy Dipole Resonance (PDR). The PDR is associated with the oscillation of a neutron skin against the core. This mode is observed below the wellknown Giant Dipole Resonance (GDR); it is present in many isotopes with a considerable neutron excess and carries a few percent of the isovector energy-weighted sum rule (see Fig. 8). Its presence has also been established for stable nuclei with a large neutron excess, like ${ }^{208} \mathrm{~Pb}$, but it is expected to be more pronounced in nuclei further from stability. The PDR strength is connected to the properties of the neutron skin, which in turn are used to constrain the equation of state of neutron-rich nuclear matter. Finally, the PDR strength plays a role in $r$-process nucleosynthesis. The presence of a neutron or proton skin also affects excitations of other multipolarities. In particular, a concentration of low-energy electric quadrupole strength, located much below the Isoscalar Giant Quadrupole Resonance, was identified in an early AGATA 

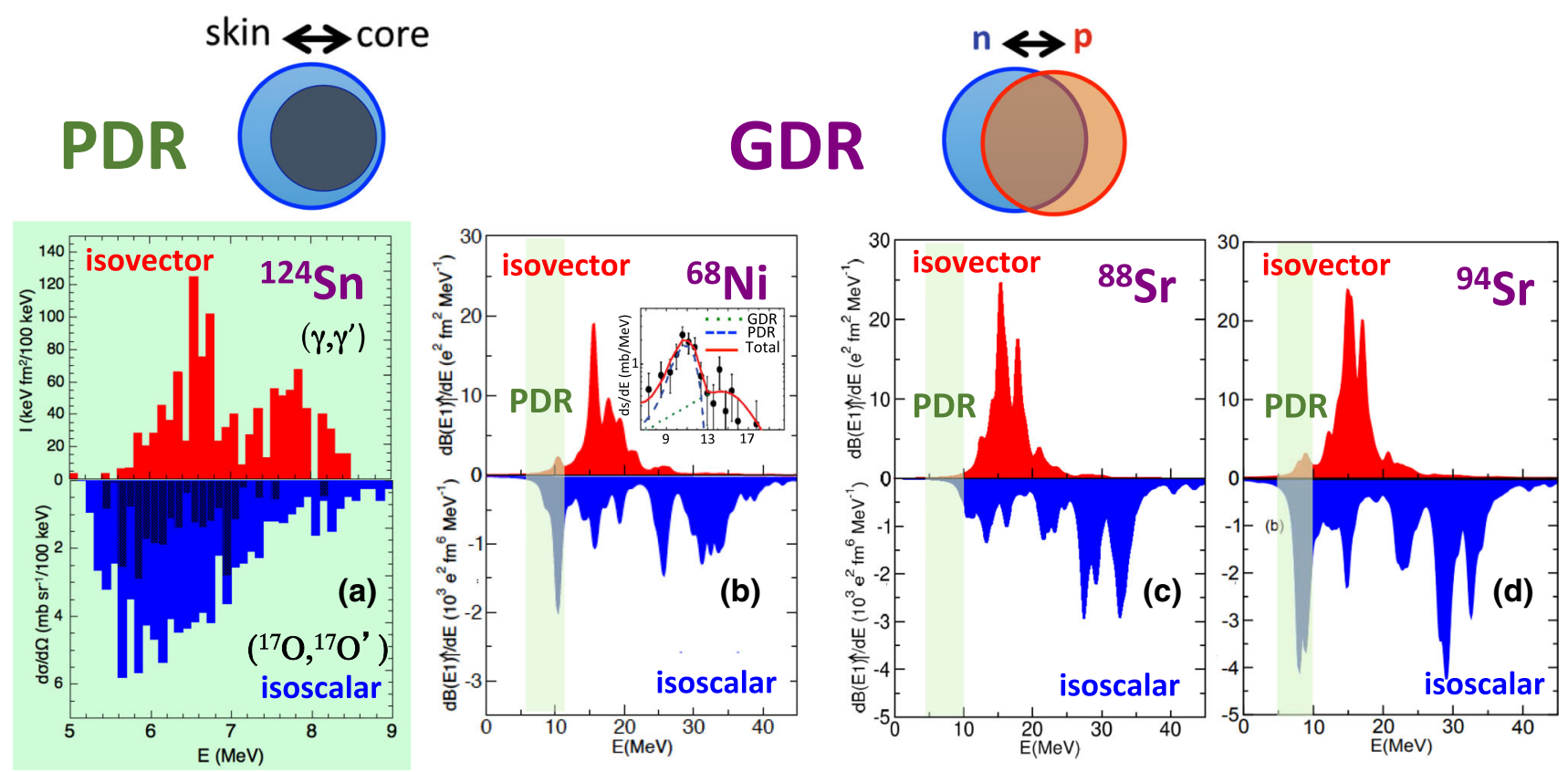

Fig. 8 a Photon decay of the PDR in ${ }^{124}$ Sn following photon scattering at TU Darmstadt (top) and heavy-ion inelastic scattering (bottom), as measured with the AGATA Demonstrator array at LNL [23]. Random Phase Approximation calculations of the strength distributions are shown for ${ }^{68} \mathrm{Ni}$ (b), for stable ${ }^{88} \mathrm{Sr}$ (c) and unstable ${ }^{94} \mathrm{Sr}$ (d) and for

experiment as a new mode of nuclear excitation known as the Pygmy Quadrupole Resonance (PQR).

Experiments to study or search for these resonances with AGATA would be performed with RIBs (both at Coulomb barrier and relativistic energies), using inelastic scattering reactions, e.g., $\left(\alpha, \alpha^{\prime}\right)$ and $\left(p, p^{\prime}\right)$, in inverse kinematics. The strong discovery potential of AGATA for these studies is based on the excellent efficiency up to very high $\gamma$-ray energies and the excellent position resolution required to correct for the expected strong Doppler effects.

\subsection{Very heavy and super-heavy nuclei}

Understanding the stability and synthesis of super-heavy nuclei (SHN) must go hand in hand with studying the nuclear structure of very heavy nuclei with $Z \approx 100$, and the reaction mechanisms leading to their production. The production cross sections of these transfermium nuclei are sufficiently high to carry out detailed spectroscopy studies. The objectives are:

- to study the collectivity of the nuclei through lifetime measurements, in particular around the islands of large deformation $\left({ }^{254} \mathrm{No}\right.$ and $\left.{ }^{270} \mathrm{Hs}\right)$,

- to determine the properties of the single-particle configurations in odd-mass nuclei, isovector (top) and isoscalar (bottom) responses. A clear increase is seen in the 5-10 MeV energy region (marked in green) for the PDR in ${ }^{68} \mathrm{Ni}$ and ${ }^{94} \mathrm{Sr}$. AGATA will extend these studies to unstable nuclei using RIBs, following the pioneering investigation performed at GSI on ${ }^{68} \mathrm{Ni}$ (inset of panel b from [24]) and ${ }^{64} \mathrm{Fe}$

- to study the role of $K$-isomerism with respect to the stability of the heaviest nuclei,

- to estimate the height of the fission barriers as a function of neutron and proton number

All these studies will tremendously profit from the availability of AGATA in conjunction with a highly efficient recoil separator with excellent beam-suppression characteristics. High count-rate capabilities are necessary in order to accept high beam intensities to counterbalance the low cross sections. The high efficiency and excellent energy resolution will allow $\gamma \gamma$ coincidence spectroscopy for the first time and in this way to disentangle the level schemes of oddmass nuclei or those built on $K$-isomers. Finally, the excellent position resolution will facilitate lifetime measurements using Doppler-shift techniques and the determination of level spins and parities using angular correlation and linear polarisation measurements, respectively.

\subsection{Spectroscopic studies for nuclear astrophysics}

Nuclear astrophysics requires careful and dedicated investigations of a large variety of nuclear processes as indispensable tools for modelling stellar evolution and nucleosynthesis. Nuclear reaction cross sections and other nuclear properties are fundamental inputs to understand the structure, evo- 
lution, and composition of a large variety of cosmic objects, including the Solar System. To determine them, we must explore the interactions between atomic nuclei in the laboratory, from stable species to very rare isotopes.

A typical measurement with a high intensity beam of light ions delivered onto a solid target would strongly benefit from the use of AGATA. Using radioactive ion beams, these studies can be extended to reactions populating more exotic species near the proton and neutron driplines, e.g., ${ }^{10,11,15,16} \mathrm{C}$, $12,13,16,17 \mathrm{~N}$ or ${ }^{14,15,19,20} \mathrm{O}$. Their decay properties and structure are mostly unknown, but may play a role in nucleosynthetic and explosive scenarios. Even less exotic C, N, and $\mathrm{O}$ isotopes are of great interest for understanding, for example, the isotopic composition observed in giant stars of various masses. For these studies, reactions between light and medium-mass nuclei, such as ${ }^{12,13,14} \mathrm{C}$ on ${ }^{9,10} \mathrm{Be}$ or ${ }^{10,11} \mathrm{~B}$ can be used.

Another important physics case is provided by the investigation of the nucleosynthesis path of the radioisotope ${ }^{26} \mathrm{Al}$ $\left(T_{1 / 2}=7.17 \cdot 10^{5}\right.$ a) with its low-lying isomeric $0^{+}$state. In particular, the cross section of the reactions ${ }^{26} \mathrm{Al}(n, p)^{26} \mathrm{Mg}$ and ${ }^{26} \mathrm{Al}(n, \alpha)^{23} \mathrm{Na}$ depend strongly on the population of the isomer, which puts constraints on the birth of the solar system and the chemical evolution of the Galaxy. The use of AGATA will allow to determine for the first time the contribution of excited states of the nuclei involved.

Heavier elements beyond the $\mathrm{Ni}-\mathrm{Fe}$ region are thought to be synthesized in Nature through the rapid neutron-capture process, or $r$-process. It is expected to take place in violent stellar events, such as neutron-star mergers or possibly core-collapse supernovae. It has been shown that even elements with $Z \approx 100$ and above (with $N \approx 170-220$ ) could be produced in the $r$-process, in particular for "cold" conditions $(T \approx 0.1 \mathrm{GK})$. Preliminary calculations of $(n, \gamma)$ reaction rates for fermium nuclei with and without the $M 1$ scissors mode in the $\gamma$-strength function $(\gamma-\mathrm{SF})$, have been performed. They show a significant impact on the astrophysical rates, also for nuclei that are accessible in the laboratory by means of fusion-evaporation experiments. Moreover, an enhancement of the reduced average $\gamma$-decay probability would boost the $\gamma$-decay channel relative to fission with subsequent consequences for the survival of heavy nuclei produced in heavy-ion reactions. Such detailed studies in nuclei produced with tiny cross sections require a $\gamma$-ray spectrometer providing not only the highest resolution and efficiency, but also capable of measuring angular correlations and linear polarisation, which are intrinsic properties of AGATA.

However, a direct measurement of the reactions of interest is often prevented by the extremely small cross sections or by the low intensity of the radioactive beams or even by the absence of appropriate radioactive targets. In this context, indirect techniques provide a valid alternative solution to estimate astrophysical rates. One such approach for neutron-

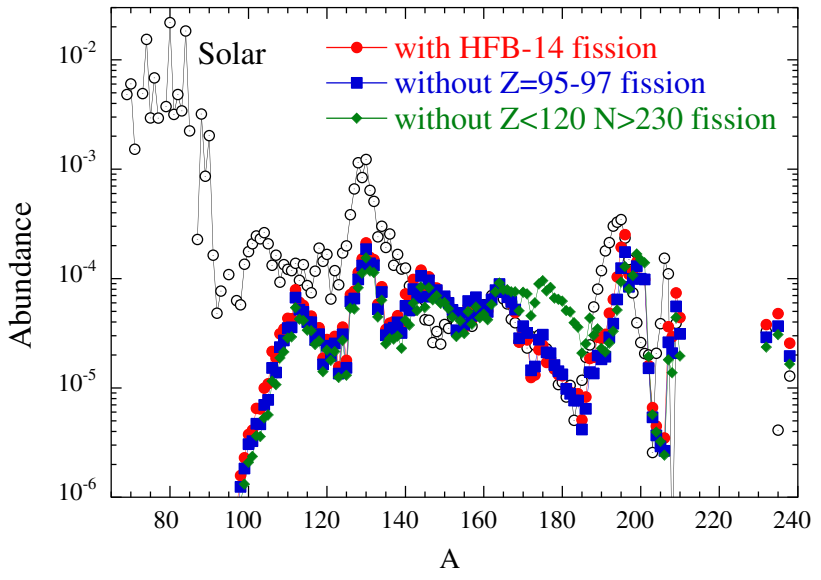

Fig. 9 Solar abundances compared with final $r$-process abundances in ejected matter from a 1.35-1.35 solar mass neutron star merger calculated with HFB-14 fission barriers (red symbols), assuming $Z=95-97$ and $N>184$ nuclei are not affected by fission (blue symbols) and assuming $Z<120$ and $N>230$ nuclei do not undergo fission (green symbols) (reproduced from [25])

capture reactions is to transfer a neutron using a $(d, p)$ reaction to the same compound level (or levels) that would be populated in the $(n, \gamma)$ reaction and then to measure the subsequent decay. Using the $(d, p \gamma)$ reaction on exotic $r$-process nuclei and performing precision measurements of the $\gamma$-ray branching ratios with AGATA will allow to determine the cross section to populate the states of interest. This so-called surrogate method relies on the assumption that the decay of the compound nucleus is independent of the entrance channel and has already been tested for low neutron energies (see also Sects. 3.3.2 and 3.5.7).

Finally, fission of very neutron-rich heavy nuclei is a key ingredient for modelling the $r$-process nucleosynthesis since it determines the final $r$-process abundance distribution (see Fig. 9). Whether or not $Z>110$ elements are produced in the $r$-process and can be found in cosmic rays or terrestrial matter also depends on the fission properties of these nuclei. As discussed in Sect. 3.4.4, AGATA in its final configuration used as a $4 \pi$ calorimeter will allow the fission barrier to be determined much more precisely than the presently used combination of $\mathrm{Ge}$ and scintillator arrays.

\section{Opportunities at future host laboratories}

Over the next decade, a number of new and enhanced accelerator facilities for nuclear physics will become available in Europe. These are described in the 2017 NuPECC Long Range Plan [18]. The major new RIB facilities FAIR/SuperFRS (Germany), SPES (Italy), SPIRAL2 (France) and HIEISOLDE (CERN) present an unrivalled opportunity to study the properties of nuclei far from stability. In addition, high- 
intensity stable beams available from, for example, GANIL (France), LNL (Italy), and JYFL (Finland) are still of major importance for nuclear structure studies. Key to these studies are advanced experimental techniques, including highprecision and high-efficiency $\gamma$-ray detection. All the major European accelerator facilities have expressed interest to host AGATA in the coming decade and beyond.

The AGATA spectrometer is by design a multi-purpose spectrometer suitable for such diverse environments as encountered at these facilities, ranging in beam energy from a few $\mathrm{MeV} / \mathrm{u}$ to beyond $1 \mathrm{GeV} / \mathrm{u}$. AGATA moves between laboratories in order to take full advantage of the different beams and facilities available, and hence to maximise the breadth of science that is addressed. Besides a wide choice of spectrometers and separators available at these host laboratories, the AGATA collaboration has also access to a wide range of complementary detectors, most of which are also travelling detectors: charged-particle detectors for tagging (DIAMANT [26], EUCLIDES [27], MUSETT [28], TRACE [29]) and for spectroscopy (MUST2 [30], GRIT [31]), neutron detectors (NEDA [32], NWALL [33,34]), $\mathrm{LaBr}_{3}(\mathrm{Ce})$ scintillators for fast-timing measurements (FATIMA [35]) or high-energy $\gamma$ ray spectrometers (PARIS [36]) and others.

This chapter describes the future opportunities making use of the existing accelerator infrastructure including ongoing major upgrades. There naturally exists some overlap in certain physics cases across the host sites. The specific facilities and beams at each site provide complementary approaches to address the key science questions.

\subsection{AGATA at the FAIR/Super-FRS facility}

The Facility for Antiproton and Ion Research (FAIR) [11] is the only facility worldwide that will deliver high-intensity RIBs, in either the ground or an isomeric state, covering the entire chart of nuclides and with relativistic energies as high as $1.5 \mathrm{GeV} / \mathrm{u}$. These beams are produced in projectile fragmentation and fission reactions, separated and identified in the Super-Fragment Separator (Super-FRS) [12] and directed onto a secondary target. FAIR is therefore a unique place to perform experiments, which take advantage of

- highest energy/velocity of the RIBs (beyond $1 \mathrm{GeV} / \mathrm{u}$ ),

- RIBs of all elements up to U,

- isotopically pure secondary beams,

- electron-free beams (fully stripped),

- isomeric beams (down to $\mu \mathrm{s}-\mathrm{ns}$ lifetimes).

AGATA offers not only a high $\gamma$-ray efficiency and an excellent peak-to-total ratio, two properties which combined lead to a high sensitivity, but furthermore it features an unprecedented position resolution due to its fine segmentation combined with pulse-shape analysis techniques. It is in particu- lar this excellent position resolution, allowing for an accurate Doppler correction of the energies of $\gamma$ rays emitted by nuclei moving with velocities reaching $50-80 \%$ of the speed of light, which will enable to take full advantage of the available high-energy RIBs. The drastically improved energy resolution, in comparison to the previous $\gamma$-ray arrays at GSI (RISING, PreSpec), but also $\gamma$-ray spectrometers based on scintillators currently used at competing laboratories, e.g., DALI2+ at RIKEN, will increase the sensitivity for many experimental studies and in some cases even enable them for the first time:

- studies of excited states in nuclei which can be produced only with very low rates (a few particles per second), thus giving access to very exotic species,

- investigation of non-yrast states at excitation energies of up to $30 \mathrm{MeV}$ and excitations built on isomeric states,

- lifetime measurements using novel high-velocity Doppler-shift techniques,

- analysis of $\gamma \gamma$ coincidences for the elaboration of complex excitation schemes, in particular for odd-mass nuclei with high level density.

The combination of high-energy heavy rare isotope beams with AGATA and the possibility of heavy secondary reaction products identification is unique for FAIR. This will enable a broad range of secondary reactions to be employed to study in particular heavy neutron-rich isotopes around the shell closure at $N \sim 126$, which so far are very difficult to access. The aim to study nuclei in this region is to explore the possible shell evolution in the region and its consequences on the astrophysical $r$-process path. This particular aspect of nuclear structure, as one of the three main motivation themes for the physics program of the whole NUSTAR (NUclear STructure, Astrophysics, and Reaction) collaboration at FAIR, is presently considered as indispensable for understanding the existence and structure of the heaviest nuclei.

To populate excited states in the nuclei of interest, the high-energy RIBs impinge on stable solid or liquid targets, which due to the high energy of the beams can be very thick thus providing high luminosities. High- $Z$ targets allow for electromagnetic excitation (Coulomb excitation), while reactions mediated by the strong nuclear force, such as inelastic scattering and knockout reactions, dominate when the heavy RIBs interact with low- $Z$ targets $(\mathrm{H}, \mathrm{He}, \mathrm{Be}, \mathrm{C}$, etc.). The different and often complementary characteristics of these reactions enable a broad range of physics topics to be addressed. Independent on the reaction mechanism used to populate the excited state of interest, the accurate determination of the $\gamma$-ray emission angle enabled by the superb position resolution of AGATA permits the development of a variety of novel experimental techniques, which take advantage of 
Fig. 10 AGATA will be the central part of the HISPEC experiment [37] located in the low-energy branch of the Super-FRS [12] and in front of the low-energy buncher (LEB) spectrometer

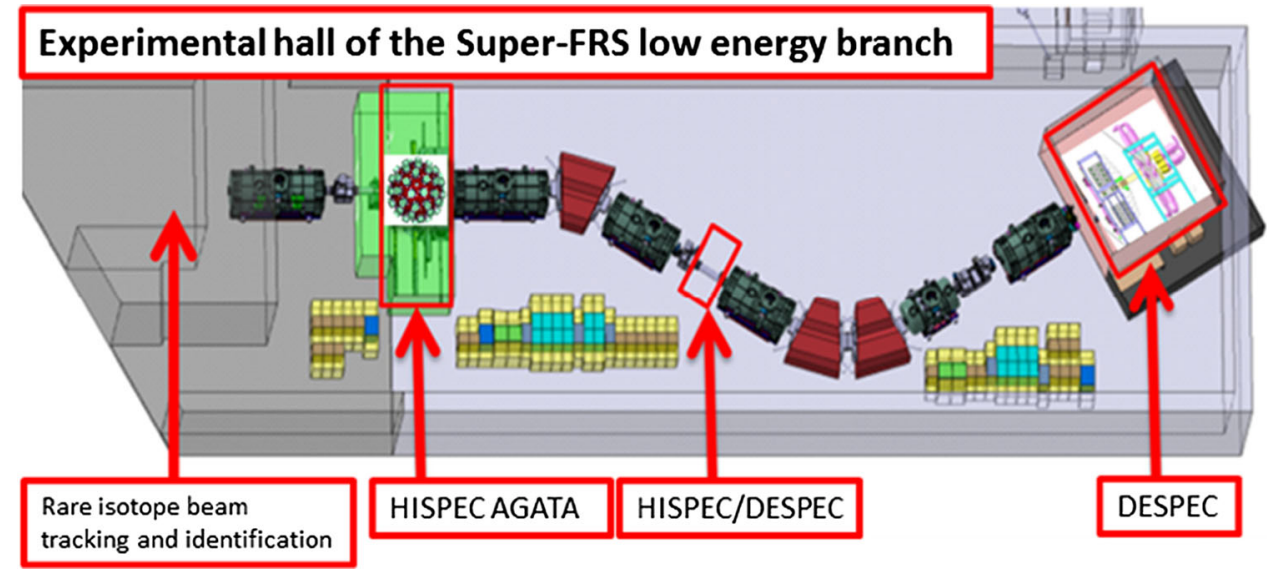

the Doppler effect to determine excited-state lifetimes. Such direct and model-independent lifetime measurements constitute an important complement to other experimental techniques.

AGATA will be located in the low-energy branch of the Super-FRS in front of the low-energy buncher (LEB) spectrometer (see Fig. 10). A core device for the reconstruction of the secondary products following both Coulomb excitation, direct or fragmentation reactions at relativistic energies is the LYCCA calorimeter [38], which, using a combination of $\mathrm{Si}$ and CsI detectors in telescope configuration, convey information on the mass number $(A)$ and charge $(Z)$ of the outgoing ions.

In the following, the main characteristics of the different techniques, which will be employed with AGATA at FAIR, will be discussed, and typical physics cases will be mentioned.

\subsubsection{Coulomb excitation to probe the electromagnetic response of exotic nuclei}

At relativistic beam energies electromagnetic excitation occurs only as a one-step process due to the very short interaction time. Consequently, only low-spin states can be excited with reasonable cross section via the absorption of virtual photons of multipolarity $E 1, M 1, E 2$, or $E 3$. The cross section for the excitation of low-lying $2^{+}$states rapidly drops with increasing beam energy, so that for the Coulomb excitation of yrast states usually energies not higher than $150 \mathrm{MeV} / \mathrm{u}$ are chosen. The excitation probability increases with beam energy for higher-lying states. This makes FAIR a unique place to measure transition probabilities from the ground and isomeric states to low-lying excited states in heavy nuclei, as well as to study Giant and Pygmy resonances, i.e., collective states at excitation energies from roughly 8-30 MeV.

Using the technique of Coulomb excitation at energies around $150 \mathrm{MeV} / \mathrm{u}$ both the single-particle structure as well as the evolution of quadrupole and octupole collectivity in the regions around the doubly-magic nuclei ${ }^{132} \mathrm{Sn}$ and ${ }^{208} \mathrm{~Pb}$ can be studied. Similarly, in regions of rapidly changing nuclear shapes and shape coexistence, for example around $Z=70-76$ or $N=60$, which cannot be reached at ISOL facilities, information about the shape of the nuclei can be obtained. This will contribute to the refinement of different theoretical models very far from stability. In these mass regions long-lived isomeric states are also known to exist. Their Coulomb excitation can provide information about the isomers themselves and the structures built on top of them. To study the Coulomb excitation of isomeric states, the prompt radiation will be detected in AGATA accompanied by ancillary detectors around the secondary target position. Additional Ge detectors, for example from DEGAS [39], will be placed at the final focal plane of the magnetic separator to register the $\gamma$ radiation either from long-lived isomeric states or following $\beta$ or $\alpha$ decay.

More than 50 years ago Axel and Brink formulated the hypothesis that the Giant Dipole Resonance can be built on any excited state, not only the ground state $[40,41]$. This hypothesis is commonly used in astrophysical applications and in the studies of hot nuclei, although a firm experimental proof of its validity is still missing. Recently, the Axel-Brink hypothesis was generalised by extending it to the Pygmy Dipole Resonance [42]. At FAIR the validity of the original and extended Axel-Brink hypotheses can be tested using relativistic Coulomb excitation to study the GDR and PDR built on both the ground and long-lived isomeric states of unstable nuclei. To study the GDR built on an excited isomeric state, the energy of the secondary beam has to be sufficiently high $(\sim 1 \mathrm{GeV} / \mathrm{u})$, so that the virtual photon spectrum covers the full GDR region, i.e., up to $25 \mathrm{MeV}$. The GDR strength in the $10-20 \mathrm{MeV}$ energy region will be measured by AGATA positioned at the secondary target position. DEGAS will serve to distinguish the excitations built on the ground and isomeric states by tagging on the $\gamma$ rays emitted in the decay of the isomeric state at the final focal plane. The measurement of 


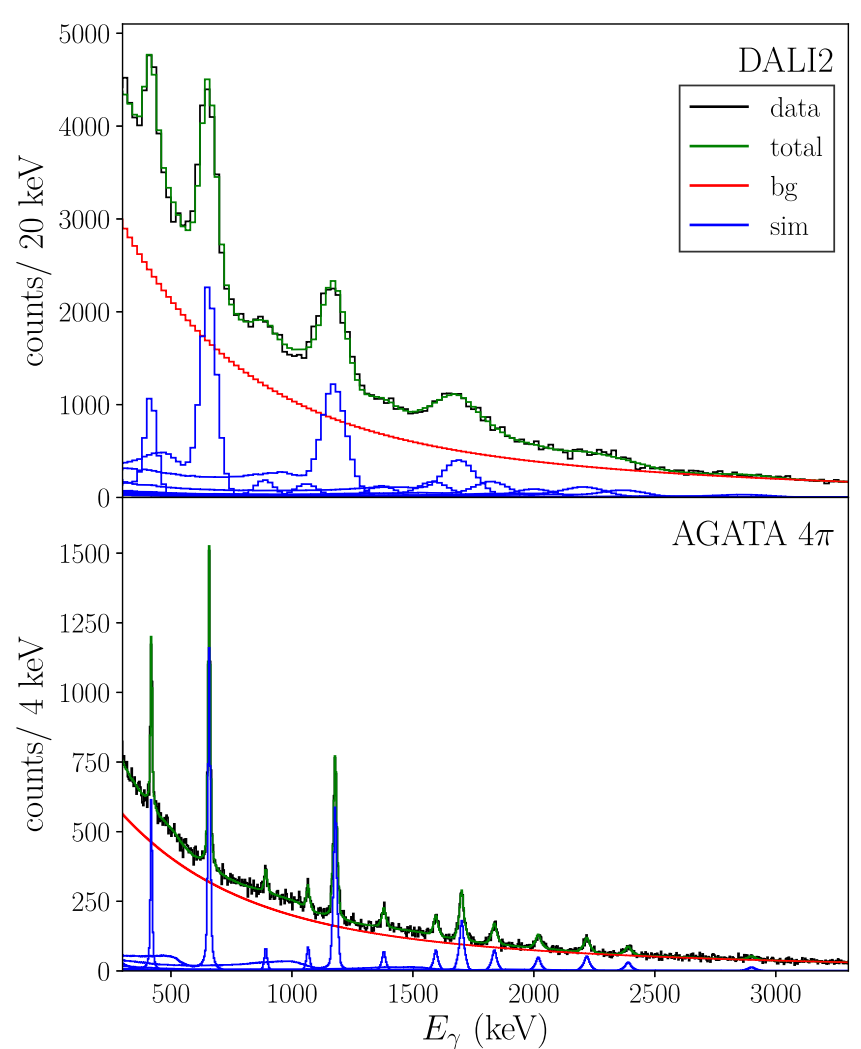

Fig. 11 (Top left) Experimental $\gamma$-ray spectrum of ${ }^{135} \mathrm{Te}$ measured with the DALI2 spectrometer following the one-neutron knockout from ${ }^{136} \mathrm{Te}$ projectiles at $165 \mathrm{MeV} / \mathrm{u}$ on a carbon target at RIKEN. (Bottom left) Simulated spectrum assuming the same reaction parameters and background shape, but using AGATA for $\gamma$-ray detection. (Right) Sim-

excitation cross sections for the inelastic scattering on low- $Z$ and high- $Z$ targets allows the separation of the isoscalar and isovector contributions. A helium target (either liquid helium or an active helium gas target) is the best choice to probe the isoscalar component of the excitation, while a gold target serves as dominant isovector probe. Long isotopic chains of semi-magic nuclei such as neutron-rich $\mathrm{Ni}, \mathrm{Sn}$, or Pb isotopes are perfect candidates for this type of studies.

At FAIR, the unique combination of the Super-FRS, AGATA, and the LEB spectrometer allows to excite highlyenergetic isomeric heavy beams up to the GDR region and disentangle the reaction products behind the secondary target. Since in neutron-rich nuclei the nature and distribution of $E 1$ strength yields valuable information about the properties of the neutron matter, its study with AGATA at FAIR will contribute to answering some of the paramount open questions in nuclear physics, namely the origin of the elements and the understanding of the nuclear equation of state.

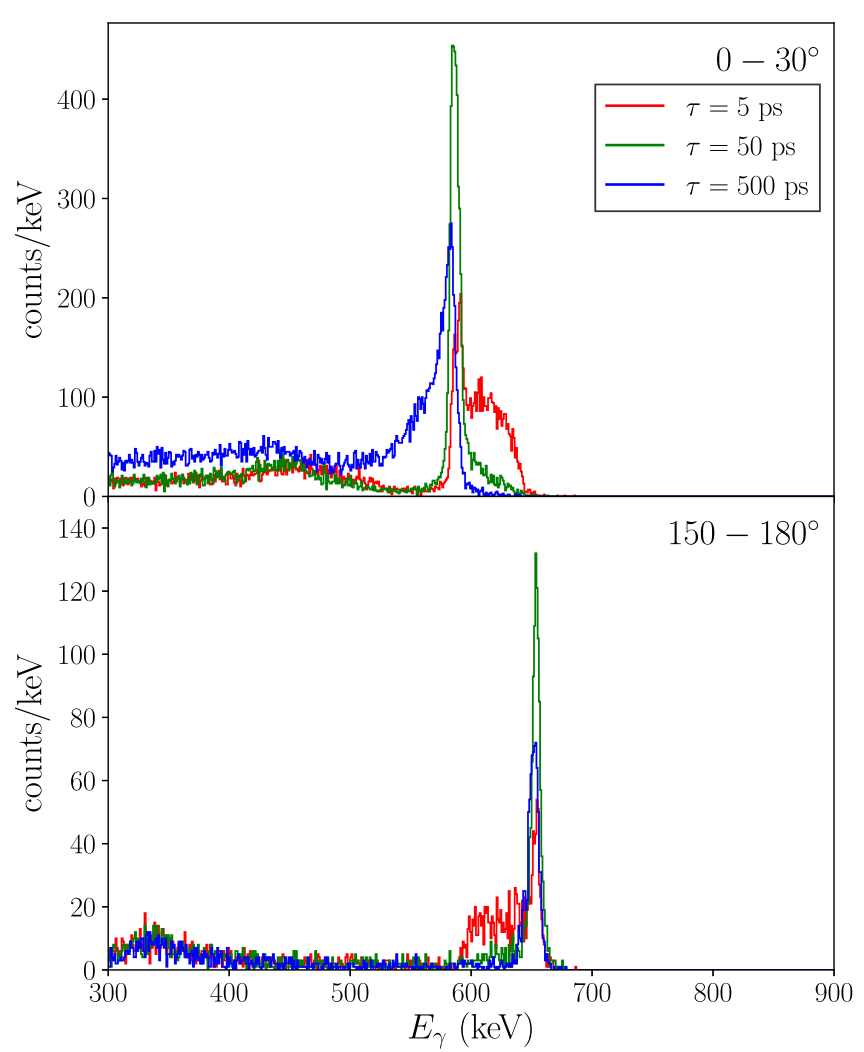

ulated line shapes of the $618-\mathrm{keV} 2_{1}^{+} \rightarrow 0_{1}^{+}$transition in ${ }^{132} \mathrm{Cd}$ emitted in forward (top) and backward (bottom) direction following relativistic Coulomb excitation at an energy of $150 \mathrm{MeV} / \mathrm{u}$ on a gold target. Lifetimes of $5 \mathrm{ps}$ (red), $50 \mathrm{ps}$ (green), and $500 \mathrm{ps}$ (blue) were assumed for the $2_{1}^{+}$state

\subsubsection{Knockout reactions and inelastic nuclear scattering}

Inelastic scattering is a powerful tool to populate excited states of exotic nuclei and, combined with electromagnetic excitation, to disentangle the contributions from proton and neutron excitations. The use of a liquid hydrogen target, being the lightest of all stable targets, combined with a vertex tracker allows an increase in the luminosity, while keeping the outstanding Doppler-correction capabilities of the AGATA spectrometer.

Knockout or other nucleon-removal reactions on light targets, on the other hand, can be used to investigate the singleparticle structure of excited states in exotic nuclei via the determination of their spectroscopic factors. In the analysis of such experiments, nuclear structure models and eikonal reaction theory are intimately connected. The direct nature of the knockout process also gives a highly selective method for the population. When coupled to shell-model calculations of the spectroscopic strength even the identification of specific states of interest in exotic species becomes possible. Twoproton knockout on the neutron-rich side and two-neutron removal on the neutron-deficient side are known to be direct, 
one-step processes, and again can be highly selective. They give access to even more exotic species as compared to onenucleon knockout reactions, while still offering manageable cross sections [43], which is determined by the two-nucleon overlap wave functions. In addition, the two-nucleon removal process can yield high spins through knockout of correlated pairs from an high- $j$ orbital coupled to maximum spin $2 j-1$.

A particularly interesting case, especially suited for experiments at FAIR, is the possibility of performing knockout reactions on isomeric beams. For example, knockout of a correlated $g_{9 / 2}$ neutron pair from known high-spin isomers in nuclei below ${ }^{100} \mathrm{Sn}$ ([44], as seen e.g., in Refs. [45,46]) will allow studying excited states of $N=Z$ nuclei up to highest spins. As this powerful technique has never been exploited before, abundant opportunities exist, as for example the $14^{+}$ isomer in ${ }^{94} \mathrm{Pd}$, which could already be investigated in the very first phase of FAIR.

In general, the importance of the unprecedented position resolution and, as a consequence, of the drastically improved energy resolution of AGATA in comparison with non-segmented $\mathrm{Ge}$ or scintillator arrays, increases with the line density in the $\gamma$-ray spectra. In the region around doublymagic ${ }^{132} \mathrm{Sn}$, for example, which is important for simulations of the $r$-process of nuclear synthesis, one-nucleon knockout from the closed $N=82$ neutron and $Z=50$ proton cores populates a variety of excited states leading to complex $\gamma$ ray spectra with a high line density (see Fig. 11). Only with the excellent energy resolution and efficiency provided by AGATA, $\gamma \gamma$ coincidence relations can be used to establish the level schemes of the populated nuclei. This enables the study of the evolution of the shell gaps as a function of proton and neutron number, challenging modern large-scale shellmodel calculations.

A particular case is the study of the region south-east of ${ }^{132} \mathrm{Sn}$, which is crossed by the $r$-process path, but is difficult to access experimentally. So far the $\gamma$ decay from excited states has only been observed in ${ }^{132}$ In following $\beta$-delayed neutron emission, and in ${ }^{132} \mathrm{Cd}$ following the two-proton knockout reaction from ${ }^{134} \mathrm{Sn}$. First information on electromagnetic properties in this quadrant could be obtained with AGATA at FAIR from a direct lifetime measurement of the $2_{1}^{+}$ state in ${ }^{132} \mathrm{Cd}$. Such a measurement, in combination with the existing experimental information for ${ }^{136} \mathrm{Te}$, would provide valuable information about neutron pairing beyond $N=82$ as a function of neutron excess. Such direct lifetime measurements only become feasible with the high energy resolution of AGATA, which allows detecting variations of the line shapes due to the velocity change during the slowingdown of the ions in the target, and the change of the emission position after the ions have left the target. These processes serve as a clock and thus allow determination of the excited state lifetime from a careful comparison to simulated line shapes (see Fig. 11). However, detectors under both forward and backward angles, and ideally a close-to- $4 \pi$ coverage, are needed in cases where neither the lifetime nor the energy of the state is known.

Similar experiments for the $N=126$ isotones are unique for AGATA and FAIR. Thanks to the angular resolution offered by AGATA, an unprecedented energy resolution is obtained for the Doppler-corrected spectra. FAIR will be the only facility where such heavy radioactive nuclei produced in few-particle removal reactions at relativistic energies can be unambiguously identified.

\subsection{AGATA at the GANIL/SPIRAL facility}

The physics opportunities with AGATA at the GANIL facility [15] span the fields of nuclear structure, nuclear dynamics, and nuclear astrophysics using heavy-ion beam collisions. The main strengths of GANIL for high-resolution in-beam $\gamma$-ray spectroscopy are the availability of high-intensity stable beams from $\mathrm{C}$ to $\mathrm{U}$ at energies ranging from the Coulomb barrier to the intermediate regime (50-100 MeV/A) delivered by the cyclotron complex, the possibility to use radioactive beams from the LISE fragment separator at intermediate energies, and post-accelerated RIBs at the Coulomb barrier from the SPIRAL1 ISOL facility.

The preferred location of the AGATA $4 \pi$ system at GANIL is in the current experimental halls, either at the target position of the VAMOS++ spectrometer [47,48], coupled to the NEDA-DIAMANT setup $[49,50]$ or at the focal plane of the LISE fragment separator as indicated by the black arrows in Fig. 12. In the long term, even more interesting opportunities will arise from post-accelerated nuclei produced by fusion-evaporation and selected in a gas-cell system at the low-energy branch of the new S3 spectrometer or, eventually, by fission fragments from SPIRAL2 Phase 2.

GANIL provides the magnetic spectrometer VAMOS which can be used either in vacuum or gas-filled mode [51,52] and the LISE separator [53]. With the VAMOS magnetic spectrometer it is possible to identify and reconstruct the kinematics of heavy ions in binary collisions up to $Z \sim 60$ and $A \sim 200$. With VAMOS in the gasfilled mode fusion-evaporation residues can be separated from the beam- and target-like projectiles and be tagged by their characteristic radioactive decays using the MUSETT detector array. The NEDA neutron detector array and DIAMANT charged-particle detector system allow tagging of residues from fusion-evaporation or transfer reactions by their neutron-proton- $\alpha$ evaporation. With GRIT the kinematics of light particles can be reconstructed after few-nucleon transfers. This rich variety of detectors makes it possible to investigate the structure of nuclei ranging from light to heavy species and from neutron deficient to neutron rich. In addition, nuclear reaction mechanisms can be studied using exotic nuclei in different energy regimes. 
Fig. 12 Possible locations for AGATA $4 \pi$ at GANIL [15] making use of both stable beam from the coupled-sector cyclotrons (CSS1/2) and radioactive beams from SPIRAL1/CIME

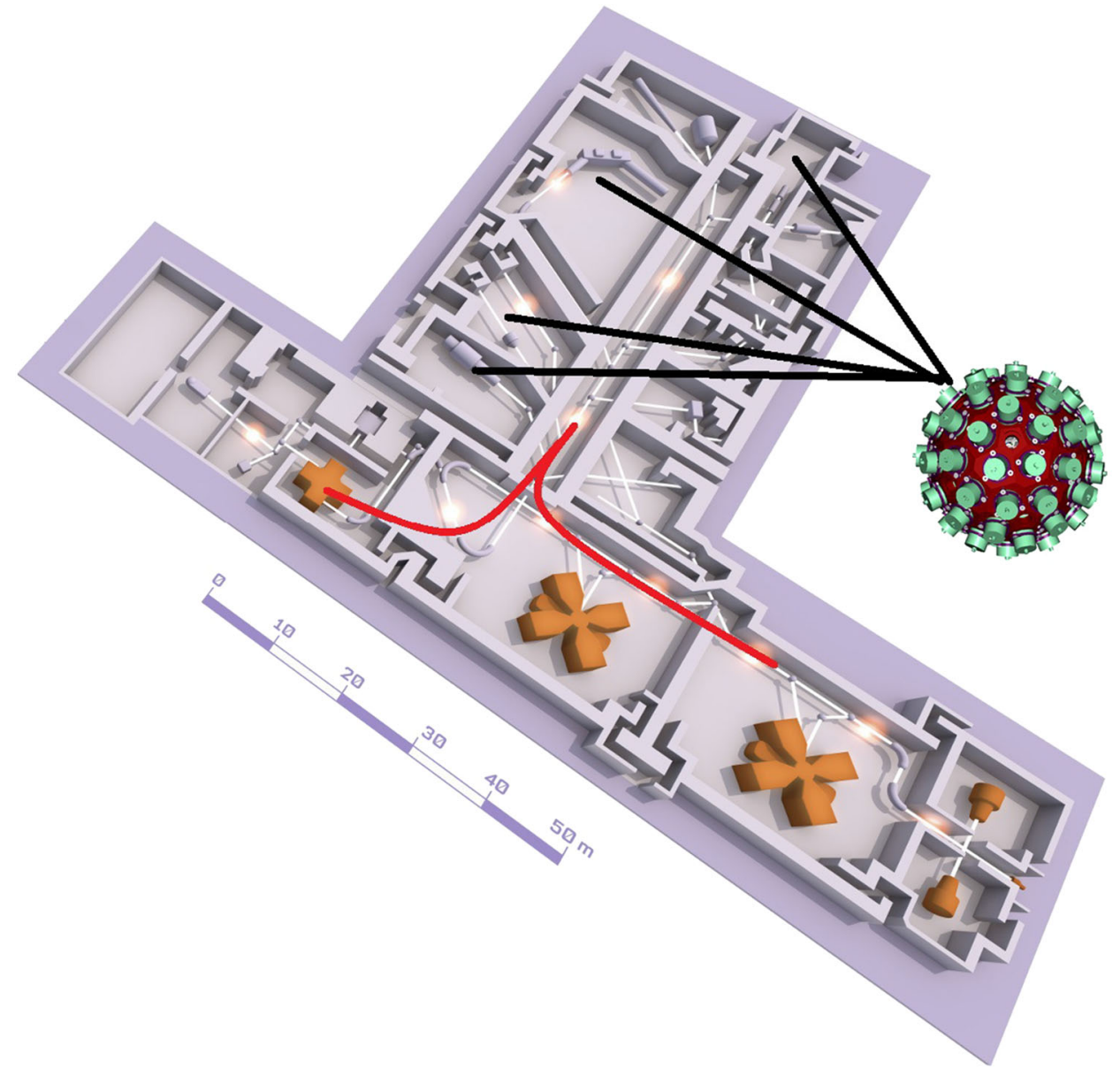

formed with very low beam intensities (down to $1000 \mathrm{pps}$ ), but require highly efficient $\gamma$-ray spectrometers with high position sensitivity. AGATA combined with state-of-the-art light charged-particle arrays will make experiments feasible aimed at determining the contributions of protons and neutrons to specific nuclear excitations.

As an example, post-accelerated beams of doubly-magic ${ }^{56} \mathrm{Ni}$ and neighbouring isotopes at $>10^{3} \mathrm{pps}$ will allow an extensive experimental program using Coulomb excitation and direct transfer reactions with ${ }^{2} \mathrm{H},{ }^{3} \mathrm{H}$, or ${ }^{3} \mathrm{He}$ targets to be carried out: including $1 n$ and $2 n$ transfer to probe the $N=28$ neutron shell closure, $2 p$ transfer to probe the $Z=28$ proton shell closure, and ${ }^{2} \mathrm{H}$ transfer to study pn pairing. Such a set of transfer reaction data would be unique. A large set of transition probabilities, spectroscopic factors and spectroscopic quadrupole moments in yrast and non-yrast states in ${ }^{56} \mathrm{Ni}$ and neighbouring isotopes will give new information on nucleonic correlations in this mass region. Using lighter elements, between $\mathrm{He}$ and $\mathrm{Ca}$, direct reactions and chargedparticle and $\gamma$-ray spectroscopy will allow low-lying bound states and their coupling to the continuum to be studied. In-beam spectroscopy of post-accelerated beams of ${ }^{6-8} \mathrm{He}$, ${ }^{8,9} \mathrm{Li},{ }^{11} \mathrm{Be},{ }^{15} \mathrm{C},{ }^{16-18} \mathrm{~N},{ }^{23-25} \mathrm{Ne}$, and ${ }^{25,26} \mathrm{Na}$ could be per- 


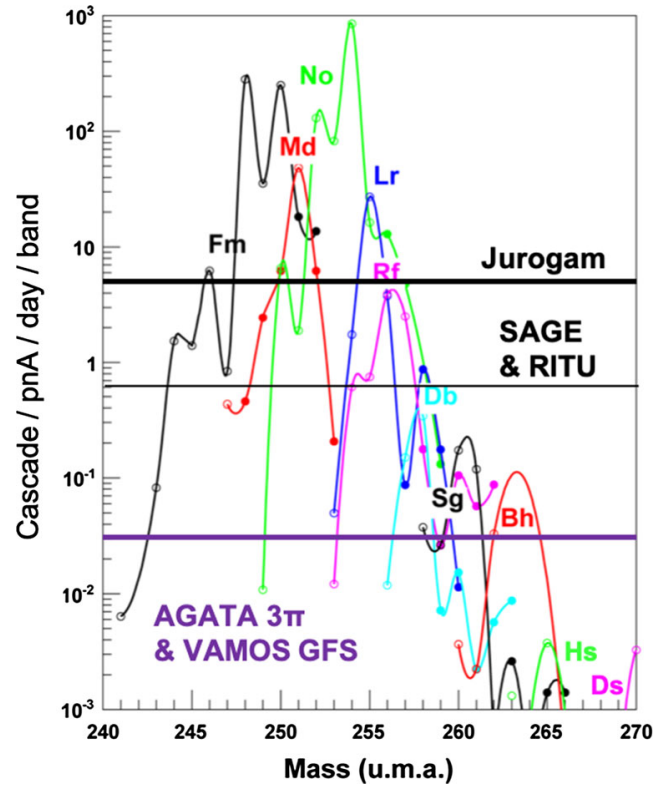

Fig. 13 (Left) Limit of sensitivity of AGATA for spectroscopy experiments on very heavy and super-heavy elements. (Right) Simulated spectrum obtained with 15 AGATA Clusters under backward angles in a plunger lifetime measurement of ${ }^{254}$ No using inverse kinematic

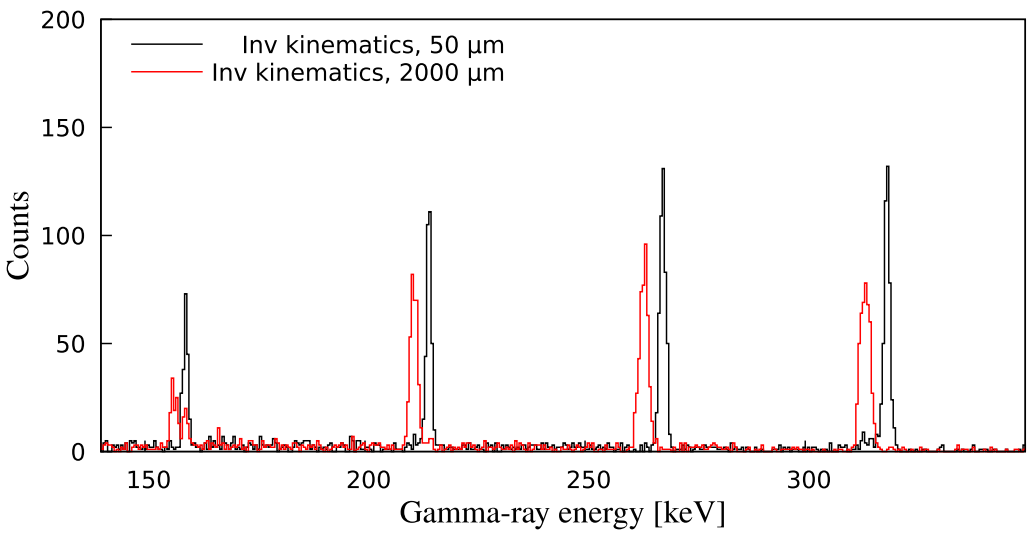

$\left({ }^{208} \mathrm{~Pb}+{ }^{48} \mathrm{Ca}\right)$ for target-to-degrader distances of $50 \mu \mathrm{m}$ and $2 \mathrm{~mm}$. Thanks to the high quality of the Doppler correction in AGATA, lifetimes in states above the $6^{+}$state could be measured

with a device for prompt electron spectroscopy would be of great benefit. The proposed experiments include the in-beam spectroscopy of nuclei as close as possible to the doublymagic deformed ${ }^{270} \mathrm{Hs}$ and the first lifetime measurement in a transfermium element in the rotational ground-state band of ${ }^{254}$ No. Figure 13 shows the expected limit in prompt $\gamma$-ray spectroscopy as a function of the AGATA solid angle coverage and transfermium masses. Approaching ${ }^{270} \mathrm{Hs}$ would be possible with the $3 \pi$-AGATA system coupled to VAMOSGFS.

As shown in Fig. 13 the very first lifetime measurement in a transfermium nucleus could be performed making use of the specific capabilities of AGATA and the ${ }^{208} \mathrm{~Pb}$ beam from GANIL. Finally, entry distribution $\left(E^{*}, I\right)$ measurements of very heavy nuclei could be performed using the AGATA $4 \pi$ array [55]. These allow the measurement of fission barriers on the condition that the neutron separation energy is larger than the saddle point. Such measurements are also important to understand the reaction mechanisms leading to the production of these very rare nuclei and, by comparing experiment with theory, will provide a better modelling of the processes involved. It may also be possible to compare the entry distributions for a given nucleus produced in standard fusion-evaporation and in multinucleon transfer reactions. This, together with cross-section measurements would certainly give a better understanding of the reaction processes. 


\subsubsection{Isospin symmetry studies}

The concept of isospin symmetry is expressed most clearly in the nuclei with conjugate neutron and proton numbers known as "mirror nuclei", having a very similar structure [56]. The breaking of the isospin symmetry by the Coulomb force increases with $Z$ and for a given mass it is at a maximum for $N=Z$ nuclei. Consequently, studies of the heaviest possible nuclei with $N \approx Z$ are of fundamental interest. Inbeam spectroscopy of the ${ }^{75} \mathrm{Sr} /{ }^{75} \mathrm{Rb}$ or ${ }^{79} \mathrm{Zr} /{ }^{79} \mathrm{Y}$ mirror pairs are examples that are accessible with AGATA at GANIL. For less exotic nuclei, electromagnetic properties of the corresponding states in mirror nuclei are a powerful tool to test isospin symmetry. Here, lifetime measurements in $A=71$ or $A=67$ nuclei are ideal cases. These could be performed using plunger techniques following fusion-evaporation reactions or multinucleon transfer. The isospin symmetry breaking can be also studied by observing the isospin mixing of nuclear states (see Sect. 2.5). In fact, every nuclear state is expected to contain, in addition to the main component of isospin $T$, minor components of different isospin. Isospin mixing can be directly accessed by measuring, for example, the isospin forbidden $(\Delta T=0) E 1$ transitions in $N=Z$ nuclei. Lifetime measurements of excited states de-exciting through $E 1$ transitions in heavy $N=Z$ nuclei allow a direct determination of the isospin mixing. Such detailed spectroscopic information combining measurements of angular distributions, $\gamma$-ray polarization and lifetimes was so far only obtained for the $N=Z$ nucleus ${ }^{64} \mathrm{Ge}$ [57]. Similar studies in ${ }^{68} \mathrm{Se}$ and ${ }^{72} \mathrm{Kr}$ would be possible with AGATA and are of great importance to investigate the evolution of isospin mixing for higher $Z$ systems.

\subsubsection{Pairing modes}

Correlations between pairs of particles (so-called pairing) are of fundamental importance in many fields of physics involving many-body systems. In the nuclear domain, a wealth of experimental evidence has been accumulated over the years supporting the existence of neutron-neutron and protonproton pairs in the isovector $(T=1)$ pairing mode $[58,59]$. For very exotic nuclei, pairing correlations can no longer be treated as a small residual interaction, since their effect is of the same order of magnitude as the binding energy of outer nucleons generated by the remaining part of the nuclear potential. The isoscalar $(T=0)$ pairing mode, in which the intrinsic spins of nucleons are aligned, is forbidden by the Pauli principle for like-particle pairs, but possible for neutron-proton pairs, as manifested in the deuteron. Obtaining information on neutron-proton $(n p)$ pairing correlations in nuclei is a long-standing ambition in nuclear physics. For $N=Z$ nuclei, isoscalar $n p$ pairing may become significant. Several manifestations of such an isoscalar superfluid state could be tested experimentally. Transfer reactions such as $\left({ }^{3} \mathrm{He}, p\right)$ or $\left(p,{ }^{3} \mathrm{He}\right)$ are ideal probes for these studies in the same way as $(p, t)$ and $(t, p)$ for neutron-neutron pairing [60]. Indeed, systematic cross-section measurements for $n p$ transfer to the $J=1^{+}, T=0$ and to the $J=0^{+}, T=1$ states along the $N=Z$ line from one closed shell to the next can be considered an ideal probe to investigate this possible new type of pairing correlations. This can be measured by means of $\left({ }^{3} \mathrm{He}, p\right)$ reactions. Because of the experimental difficulties in studying intermediate-mass $N=Z$ nuclei, the existing information is mostly limited to $s d$-shell nuclei, with very scarce data available above the $f p$-shell nuclei. ${ }^{58} \mathrm{Cu}$ is one of the best candidates accessible experimentally as it is the only odd-odd nucleus in this region with a $J=1^{+}, T=0$ ground state (all others having $J=0^{+}, T=1$ ). The high- $j$ orbitals should favour the development of collectivity and particularly of a superfluid state. There is indeed a strongly deformed band known built on the $9^{+}$state (with $\pi_{9 / 2} \nu_{9 / 2}$ configuration) [61]. Studies towards even heavier nuclei, e.g., ${ }^{72} \mathrm{Kr}$ and ideally ${ }^{92} \mathrm{Pd}$ would be of utmost importance to investigate the evolution of pairing properties for higher $Z$ systems. Therefore, the key physics cases for AGATA at GANIL would be to study the reactions ${ }^{3} \mathrm{He}\left({ }^{72} \mathrm{Kr}, p\right){ }^{74} \mathrm{Rb}$ and ${ }^{3} \mathrm{He}\left({ }^{56} \mathrm{Ni}, p\right){ }^{58} \mathrm{Cu}$ using SPIRAL1 beams.

An alternative approach to probe the nature of pair correlations in neutron-deficient nuclei is to study the level structure of $N=Z$ nuclei at moderate to high angular momenta. The Coriolis effect acting on the paired nucleons in a deformed rotating nucleus, which drives them to align their intrinsic angular momenta along the collective rotational axis, is selective with respect to the residual interaction. In the case of normal $T=1, S=0$ pairing the Coriolis effect leads to breaking the nucleonic pairs, which produces discontinuities in the rotational behaviour (so-called "backbending" [62]). In contrast, in the case of strong deuteron-like $(T=0, S=1)$ correlations the $n p$ pairs are already spin-aligned and the Coriolis force is not expected to perturb the system as violently. Consequently, significant differences in the rotational behaviour are expected depending on whether a deformed nucleus is dominated by $T=1$ or $T=0$ pair correlations. Such phenomena can be probed experimentally using fusionevaporation reactions and $\gamma$-ray spectroscopy by observing heavy $N \approx Z$ nuclei in excited states up to moderate spins. The presence of $T=0$ pairing may slightly enlarge the pair gap at low spins producing a systematic shift in the rotational crossing frequency of the backbending effect in $N=Z$ nuclei as compared with neighbouring nuclei. This idea, first suggested for ${ }^{72} \mathrm{Kr}$, has been explored in the medium-spin region of $N=Z$ nuclei with $A=70-80$. Evidence for the shift of the crossing frequency is, however, not yet conclusive, since possible shape changes precluded a precise determination of the band-crossing frequencies. Therefore, new experimental data on heavier $N=Z$ systems, which are 
less prone to shape changes, are needed. A complete study of yrast states up to moderate angular momentum in heavier $N=Z$ nuclei towards ${ }^{100} \mathrm{Sn}$ is a major goal requiring the full performance of the AGATA tracking spectrometer (see Fig. 6).

\subsubsection{Nuclear astrophysics}

Nuclear astrophysics studies will strongly benefit from a highly efficient $\gamma$-ray tracking detector array (see Sect. 2.9) since the reaction rates in stars can be strongly influenced by the nuclear properties of the involved nuclei, thus affecting the abundance of the chemical elements.

While thermonuclear $\mathrm{H}$ and $\mathrm{He}$ capture reactions are the most important, hydrogen and helium being about $99 \%$ of the baryonic matter in the Universe, and having the lowest Coulomb barriers, some key processes are also influenced by the structure of light nuclei, such as Li, Be, B, C, O. In particular, near-threshold resonances, such as those in ${ }^{12} \mathrm{C}$ (the famous "Hoyle state") and in ${ }^{16} \mathrm{O}$ can exhibit a pronounced cluster structure that alters the reaction rates. Knowing electromagnetic decays from unbound states would represent a big step forward. However, $\gamma$-decay branching ratios are of the order of $10^{-3}$ or lower. This means that direct measurements at the relevant energies (usually of the order of hundreds of $\mathrm{keV}$ ) are strongly hindered by the extremely low cross section as well as by the background from cosmic rays and natural radioactivity. In particular, the ${ }^{12} \mathrm{C}(\alpha, \gamma){ }^{16} \mathrm{O}$ reaction is of paramount importance in the advanced burning of a massive star, but the reaction rate is still rather uncertain. As a consequence, the uncertainty on the $\mathrm{C} / \mathrm{O}$ ratio that governs the final fate of the star is quite large.

Reactions involving radioactive nuclei also play a key role in nuclear astrophysics, particularly in explosive scenarios such as X-ray bursts, novae, and supernovae. When direct measurements are not feasible, indirect approaches, such as $(d, p)$ transfer reactions, are in order to populate key astrophysical resonances and thus to obtain information on their most important spectroscopic properties, e.g., energies, spinparity values and spectroscopic factors, so that the reaction rate can be determined. However, uncertainties remain in the understanding of these phenomena for key reactions such as ${ }^{18} \mathrm{~F}(\mathrm{p}, \alpha){ }^{15} \mathrm{O},{ }^{25} \mathrm{Al}(\mathrm{p}, \gamma){ }^{26} \mathrm{Si}$ or ${ }^{30} \mathrm{P}(\mathrm{p}, \gamma){ }^{31} \mathrm{~S}$. For X-ray burst nucleosynthesis, it was shown that a few tens of reactions play an important role [63].

Nuclear states of interest for astrophysics typically exist in regions of large level density, thus identifying them from detecting charged particles alone is often impossible. Consequently, the use of a high-resolution $\gamma$-ray spectrometer has become the method of choice recently in order to simultaneously measure the excitation energy of the state of interest and detecting the $\gamma$ rays emitted from this state. As the reaction products travel with the initial beam energy $(\sim 10 \mathrm{MeV} / \mathrm{u})$, large Doppler effects occur, making a position-sensitive $\gamma$ ray spectrometer essential to provide the ultra-high resolution required to perform precision spectroscopy measurements with RIBs. Compared with current arrays AGATA will provide in addition full $\gamma$-ray tracking, improving efficiency and spectrum quality, and a large interior space to host ancillary detectors for particle detection.

\subsubsection{Nuclear structure at the highest spins}

As discussed in Sect. 2.6 many observations occurring already at moderate nuclear deformation, such as the nuclear pairing phase transition, the structure beyond the termination of rotational bands, when all single-particle spins are fully aligned, and nuclear triaxiality and chirality, deserve more in-depth studies to be fully understood. Another amazing observation is the phenomenon of the so-called "identical bands", where the $\gamma$-ray energies of superdeformed (SD) bands in neighbouring nuclei differing in mass are identical within $1 \mathrm{keV}$, i.e., less than $10^{-5}$ of the total moment of inertia. The mechanism manifests itself through vanishing differences between the kinematical moments of inertia of two different nuclei, a feature which is all but intuitive. Finally, certain SD configurations are also predicted to correspond to non-axial shapes, manifesting molecular symmetries.

Hyperdeformed structures are also predicted, but have not yet been found experimentally due to the lack of resolving power of the previous-generation $\gamma$-ray spectrometers. The population of these extremely elongated nuclear shapes is a very delicate process, which seems to be related to the presence of the nuclear Jacobi shape transition at high temperature in the compound nucleus and to the associated lowenergy component of the Coriolis splitting of the GDR. Precise theoretical predictions enable the determination of the spin feeding window of these structures, which will allow optimisation of experiments searching for hyperdeformed structures. These studies will also bring new information on how specific single-nucleonic shell structures stabilise nuclear matter on the way to fission. The experimental effort for the discovery of hyperdeformation and the Jacobi shape transition is guided by theoretical calculations identifying the best candidates. In [64], revisited predictions identified neutron-deficient $\mathrm{Cd}$ isotopes as good candidates, which can be populated in the reaction ${ }^{64} \mathrm{Ni}\left({ }^{48} \mathrm{Ca}, 3-5 \mathrm{n}\right){ }^{107-109} \mathrm{Cd}$. The Jacobi shape transition is predicted to occur, for example, in ${ }^{46} \mathrm{Ti}[65]$ or ${ }^{170} \mathrm{Yb}$ [66].

Phase transitions are among the most fascinating collective phenomena which engage significant subsets of nucleons in the system. They lead "all of the sudden" to jumping from one phase to another, e.g., from the nuclear superfluid phase, in which the nuclear moment of inertia is about $50 \%$ of the rigid-body value, to the regular phase, in which the moment of inertia takes its rigid body value. This transition 
may be induced by increasing collective rotation or, alternatively, nuclear temperature. What are the mechanisms which initiate these transitions? Why aligning one pair in a given nucleus can lead to the complete disappearance of superfluidity whereas a similar pair in another nucleus leaves the moment of inertia at $80 \%$ of the rigid-body value? Despite the success of some theoretical approaches, a deeper understanding of the phenomenon goes beyond existing models. A search for the critical frequency of the phase transition can be performed in the neutron-deficient $\mathrm{Dy}, \mathrm{Er}$ and $\mathrm{Yb}$ isotopes and other mass regions (Ba, Th, Ra, Rn) [67].

The shape instabilities or deformation softness may give rise to a dramatic shape and structure evolution with increasing spin manifested as disappearance of the nuclear rotational collectivity known as band termination. The nuclear motion at angular momenta in the vicinity of the critical spins of a Jacobi transition assumes a character of a dramatic shape instability. Indeed, the shape can oscillate between strongly oblate and prolate with elongations exceeding those observed for super-deformation, while the excitation energy changes only by a few hundred keV. Such dramatic shape transitions at no energy cost are accompanied by virtually dozens of individual nucleonic level crossings corresponding to equally dramatic intrinsic structure rearrangements. Given that various orbitals, which participate in these level crossings, have dramatically different nodal structures (spatial distributions of the particles) and the fact that all of them are accompanied by virtually negligible energy changes may indicate new states of matter and/or phase transitions, similar to those intensively studied in condensed-matter physics.

Nuclear structure studies at the highest spins are the show case for a full $4 \pi$ AGATA array. Only with the highest possible $\gamma$-ray detection efficiency combined with the tracking capabilities to minimise residual Doppler effects and to optimise the peak-to-background ratio, the necessary sensitivity can be achieved to exploit very high-fold $\gamma^{n}$ coincidences (see Fig. 7).

\subsection{AGATA at the HIE-ISOLDE facility}

The Isotope mass Separator On-Line Device (ISOLDE) facility [68] at CERN has a unique expertise worldwide in production of purified, low-energy exotic beams. The radionuclides, spanning a vast area of the nuclear chart, are produced by high-intensity $1.4-\mathrm{GeV}$ proton beam impinging on specially developed thick targets. The selective ionisation, extraction, and separation techniques have been refined over the 50 years of the continuous operation of the facility. In total, over 1300 isotopes of more than 70 elements have been delivered by ISOLDE.

Commissioned in October 2015 and subsequently upgraded, the new linear accelerator HIE-ISOLDE [13] (see Fig. 14) will bring the final energy of post-accelerated radioactive ion beams of up to $10 \mathrm{MeV} / \mathrm{A}$ and beyond $A>200$. In addition, new developments aiming at delivering more intense and isotopically pure beams are under way. Such beam energies provide an important increase of multi-step Coulomb-excitation cross sections and allow for nucleon transfer reactions on all radioactive nuclei produced at ISOLDE. The combination of the outstanding variety and quality of beams available at HIE-ISOLDE with unparalleled efficiency and energy resolution of AGATA enables broadening the scope of physics questions that can be addressed via detailed and precise studies at the heart of the most important regions of the nuclear chart.

At the beam energies of HIE-ISOLDE, AGATA provides the best energy resolution (with respect to intermediate or relativistic beam energies) thanks to the HPGe detectors and much reduced Doppler broadening due to pulse-shape analysis and $\gamma$-ray tracking techniques. The high resolution and excellent Doppler-shift reconstruction is especially beneficial in Coulomb excitation, Doppler-shift techniques (DSAM or RDDS) to measure lifetimes and transition strengths and to identify band structures. This is particularly important for studies of odd-mass nuclei, when many lines are expected in the $\gamma$-ray energy spectra, typically in a narrow energy range. When moving far from the line of stability, the nuclear half-lives become comparable with the time needed for beam separation and post-acceleration. Consequently, the resulting beams of very exotic nuclei suffer inherently from contamination due to in-flight decay. AGATA will provide higher resolving power, not only from the increased efficiency, but also from a better peak-to-background ratio and better Doppler correction for peak identification. Moreover, higher granularity will enable determination of multipolarities of observed $\gamma$-ray transitions from measurements of angular correlations and linear polarisation of $\gamma$ rays.

In transfer reactions, particle detection provides the cross sections, proportional to spectroscopic factors and related to initial and final shell occupancies. With RIBs and in inverse kinematics, there are strong limitations on the energy resolution provided by particle detection - rarely better than $300 \mathrm{keV}$. As shown in measurements performed at ISOLDE and elsewhere, the coupling to an efficient $\gamma$-ray spectrometer is in many cases essential. Efficiency is the key word here: the Miniball array, with around $\sim 10 \%$ photopeak efficiency, forces the use of relatively thick targets that compromise particle-detection resolution further, and does not provide enough statistics to analyse particle angular distributions with a condition on selected $\gamma$ decays. The $4 \pi$ AGATA set-up would open a completely different scenario by providing good statistics for particle- $\gamma$ coincidence events and, in turn, a much better background subtraction for pure particleemission events populating, for example, the ground state or isomers with half-lives longer than a few tens of nanoseconds. The high statistics would also allow to collect particle- 
Fig. 14 Possible location of AGATA on HIE-ISOLDE XT01 beam line

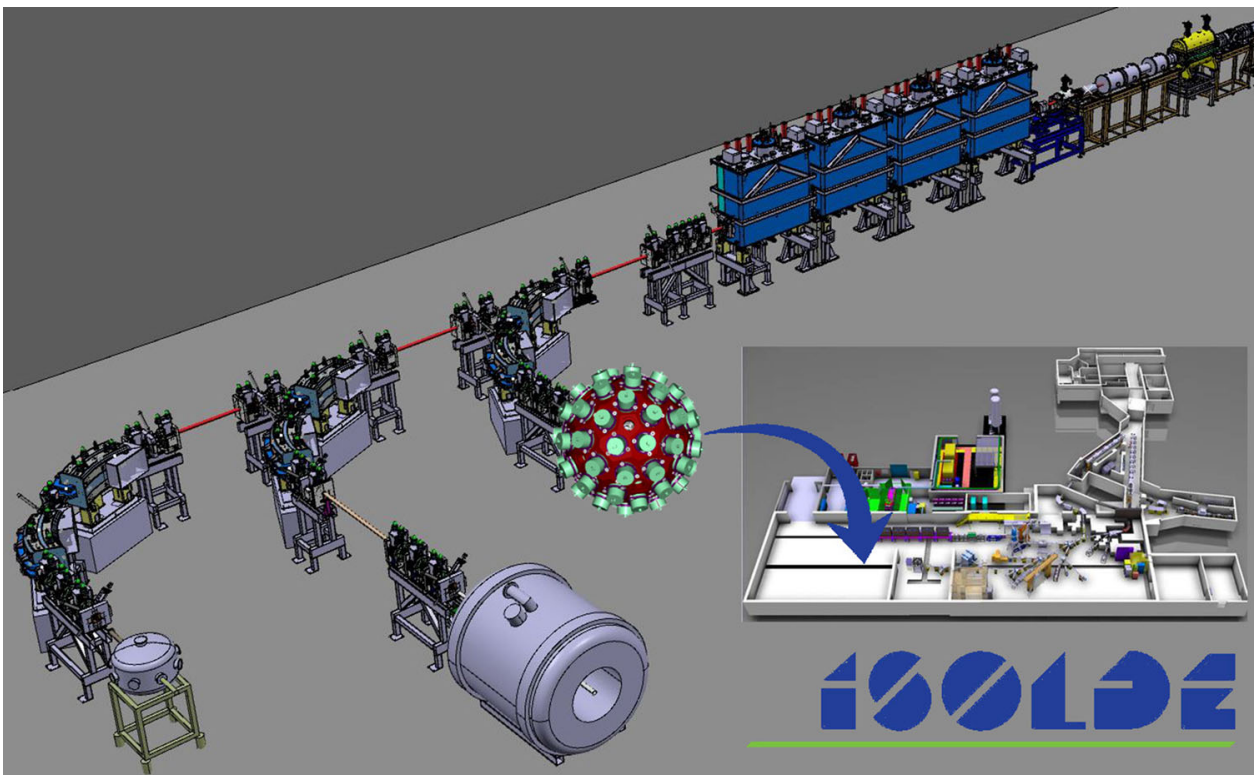

$\gamma$ angular correlations, which can be used, together with the $\gamma \gamma$ coincidences from the decay of the populated states, to constrain the spins in the product nucleus. A highly-efficient $\gamma$-ray array is especially of interest for the full reconstruction of the strengths in the product nucleus, up to energies (even of a few MeV) above the shell gap, for which the density of states becomes important.

Some key physics cases, highlighting both the uniquely available beams at ISOLDE and the state-of-the-art capabilities of AGATA are discussed below.

\subsubsection{Studies of nuclei around magic numbers}

The shell evolution, as determined by the underlying nucleon-nucleon interaction, leads to a number of remarkable effects, for example at the $Z=28$ shell closure from $N=$ $40\left({ }^{68} \mathrm{Ni}\right)$ to doubly-magic ${ }^{78} \mathrm{Ni}$. This includes the swift onset of collectivity observed below ${ }^{68} \mathrm{Ni}$ [69], determined by the gaps in the neutron-orbital $g-d-s$ sequence; the evolution of the proton $f_{7 / 2}-f_{5 / 2}$ splitting from $N=40$ to $N=50$ [70,71]; the lowering of the neutron $s_{1 / 2}$ orbital in the $N=51$ isotones from $Z=40$ to $Z=28$.

AGATA would be particularly important in identifying weak components of the strength when it is fractioned to higher-lying states. In $(d, p)$ transfer reactions, for example on $68,70,72 \mathrm{Ni}$ and possibly in Fe isotopes, the neutron- $d_{5 / 2}$ strength could be tracked, while $\left(d,{ }^{3} \mathrm{He}\right)$ transfer reactions on the even ${ }^{72-78} \mathrm{Zn}$ isotopes could determine the proton $f_{7 / 2}-f_{5 / 2}$ splitting. Further, it could provide a better characterisation of the low-lying $1 / 2^{+}$strength identified in ${ }^{79} \mathrm{Zn}$ [72] and still looked for in ${ }^{81} \mathrm{Zn}$.

In the light even-mass $\mathrm{Sn}$ isotopes a discrepancy has been found between predictions of large-scale shell-model cal- culations, using microscopically derived interactions, and experimental reduced transition probabilities between the first excited $2^{+}$state and the ground state. Measurements using low- as well as high-energy Coulomb excitation (e.g., [73-76]) display consistent results within measurement precision, but may indicate different trends towards the lighter masses. The reduced transition probabilities were also remeasured recently for the stable mid-shell nuclei, ${ }^{112-124} \mathrm{Sn}$. Surprisingly, those showed a decrease in the $B(E 2)$ values at mid-shell, contrary to earlier high-precision experiments and most model predictions. This is particularly interesting since the even Sn chain has been a textbook example for a simplified seniority scheme in the past.

This fluid situation with the new experimental results has spurred new theoretical interest in the region. Calculations now predict different trends towards ${ }^{100} \mathrm{Sn}[77,78]$, but cannot be compared with precision data as these do not exist yet for the lightest isotopes. In particular, a clear assignment of non-yrast $2^{+}$states and $3^{-}$states is important. The performance of AGATA combined with the advances in beam development will allow high-precision multiple Coulombexcitation measurements as far as ${ }^{104} \mathrm{Sn}$.

The two doubly-magic nuclei ${ }^{132} \mathrm{Sn}$ (50 protons, 82 neutrons) and ${ }^{208} \mathrm{~Pb}$ (82 protons, 126 neutrons) have the features of a classic shell-model core. The structure of neutron-rich isotopes beyond ${ }^{132} \mathrm{Sn}$ is related to the behaviour of the neutron $f_{7 / 2}$ orbital as more neutrons are added, and to the possible ensuing shell closure at $N=90$ as predicted by certain shell-model calculations with empirical interactions. The latter is similar to that of the $\mathrm{Ca}$ isotopes where the filling of the $1 f_{7 / 2}$ orbital from $N=20$ onwards leads to the $N=28$ shell closure. Future experiments with AGATA will aim to investigate, in particular, the quadrants south-east of ${ }^{132} \mathrm{Sn}$ and 
${ }^{208} \mathrm{~Pb}$, where excited states are scarcely known. For example, proton single-particle states and their interactions below $Z=82$, and indirectly the robustness of the $N=126$ closed shell, can be analysed by studying single proton-hole nuclei in the $\mathrm{Tl}$ and $\mathrm{Hg}$ nuclei with $N \geq 126$.

Another point of interest is the transition from a seniority behaviour to a collective character of excited states in nuclei when the number of valence particles increases. Such studies require a precise knowledge of the electromagnetic transition strengths for transitions de-exciting the yrast and non-yrast $2^{+}$states. Good starting points for such measurements are ${ }^{136} \mathrm{Te}$ and ${ }^{214} \mathrm{Po}$. The anomalous properties of the first $2^{+}$state of ${ }^{136} \mathrm{Te}$ are well known [79], but neither lifetimes of the other yrast states are known nor has the isovector $2^{+}$state been identified. In the case of ${ }^{212} \mathrm{Po}$, a peculiar low collectivity is observed for both the yrast $2^{+}$state and the isovector $2^{+}$state [80], which cannot be explained in the framework of the shell model. Due to insufficient knowledge of lifetimes in ${ }^{214} \mathrm{Po}$ it is not clear whether this peculiarity persists with increasing neutron number. The states of interest in both ${ }^{212} \mathrm{Po}$ and ${ }^{136} \mathrm{Te}$ can be populated in multistep Coulomb excitation or $\alpha$-transfer reactions in inverse kinematics. Their lifetimes can be measured employing differential DSAM and plunger methods. The high efficiency of AGATA and its tracking capability, which enables precise Doppler correction of the $\gamma$ rays, are of utmost importance.

\subsubsection{Reactions relevant for nuclear astrophysics}

Astrophysical X-ray bursts are thought to be the result of thermonuclear explosions in the atmospheres of accreting neutron stars in close binary systems. They are the most frequent stellar explosions to occur in our Galaxy. Detailed observations of these events have been obtained by a host of space-based X-ray observatories in recent years. Consequently, by gaining a detailed understanding of the nuclear processes responsible for the shape of the resulting light curve, it will be possible to model the dynamical impact $\mathrm{X}$-ray bursts have on the evolution of the Milky Way and will also gain significant insight into the properties of neutron stars, environments representing the extremes of nuclear matter. A number of nuclear reactions have been highlighted as having a particularly significant influence on the X-ray burst light curve which can be uniquely accessed with the suite of neutron-deficient beams available at HIE-ISOLDE. These include the waiting point nucleus ${ }^{64} \mathrm{Ge}$, whose destruction can be investigated through the $d\left({ }^{64} \mathrm{Ge}, p\right){ }^{65} \mathrm{Ge}$ reaction and the ${ }^{61} \mathrm{Ga}(p, \gamma){ }^{62} \mathrm{Ge}$ reaction, which is predicted to have a dramatic influence on the light curve - that can be studied indirectly with $d\left({ }^{61} \mathrm{Zn}, p\right)^{62} \mathrm{Zn}$ transfer.

New, indirect techniques must often be employed in order to estimate astrophysical neutron-capture rates since the cross sections cannot typically be directly measured. As dis- cussed in Sect. 2.9 one such approach is the so-called surrogate method [81] where the transfer of a neutron using a $(d, p)$ reaction leads to the same compound level (or levels) that would be populated in neutron capture. This approach has already been tested: the cross sections for the $(d, p \gamma)$ reaction on ${ }^{171,173} \mathrm{Yb}$ were compared with the known neutroncapture cross sections and the two were found to agree for neutron energies of about $100 \mathrm{keV}$ [82]. Such studies pave the way for future measurements on more neutron-rich nuclei at HIE-ISOLDE.

Furthermore, one can also exploit quasi-free transfer reactions, in a restricted phase space region, by means of the socalled Trojan Horse Method, and determine directly the cross section of the two-body reaction of interest at astrophysical energies [83-85]. In the Trojan Horse Method, these $(d, p)$ reactions are studied using deuterons as virtual neutron targets, under the assumption that the proton inside the deuteron acts as a spectator in the interaction between the neutron and the short-lived nucleus. This approach is quite unique to investigate neutron-induced reactions on short-lived nuclei, overcoming the difficult task to produce targets containing these elements $[86,87]$. With this approach one can reach the astrophysical energy region using beams in the $1-10 \mathrm{MeV} / \mathrm{u}$ energy range, since a fraction of the beam energy is used in the breakup of the participant-spectator system. The $p-n$ intercluster motion is thus used to cover the region of astrophysical interest allowing to measure the cross section in the whole relevant range very efficiently with a single beam energy.

For the techniques mentioned above, the excited states, often decaying with weak $\gamma$-decay branches, have to be identified using high-resolution $\gamma$-ray spectroscopy. The low reaction cross sections and limited exotic beam intensities will require the use of AGATA for the most interesting cases.

\subsubsection{Studies of quadrupole shapes and shape coexistence far from closed shells}

At beam energies available at HIE-ISOLDE, the probability of multi-step Coulomb excitation is enhanced, promoting population of non-yrast states. Experiments where AGATA is combined with a conversion-electron spectrometer such as SPEDE [88] will open the possibility to probe, in a single measurement, the shapes, collectivity, and location of bandhead states of the excited nucleus. The search for coexisting configurations will strongly benefit from $\mathrm{e}^{-}-\gamma$ coincidence measurements.

The neutron-deficient $\mathrm{Pb}$ region is well known for intruder configurations associated with different shapes [89]. In the Po isotopes, intruder $0^{+}$states have been observed from ${ }^{202} \mathrm{Po}$ down to ${ }^{196} \mathrm{Po}$. In-beam $\gamma$-ray data suggest the first excited state in ${ }^{194}$ Po being an excited $0^{+}$state at $\sim 230 \mathrm{keV}$ energy, making it one of the lowest such states on the whole nuclear 
chart. Similar cases with low-lying $0^{+}$states associated with intruder configurations were found in, e.g., ${ }^{186} \mathrm{~Pb}$ and ${ }^{180} \mathrm{Hg}$.

The analysis of Coulomb-excitation measurements on light $\mathrm{Hg}, \mathrm{Po}$, and $\mathrm{Pb}$ isotopes has proved to be difficult due to the lack of lifetime information. Such measurements can be realised by combining the standard Coulomb excitation setup with a plunger device, giving extra sensitivity from the independent determination of lifetimes. This leads to a more precise extraction of quadrupole moments for yrast and nonyrast states. Complementary information can be obtained from studies of odd-mass nuclei. For example, studies of coupling of the odd neutron to the even-mass core in the ${ }^{185,187} \mathrm{~Pb}$ isotopes can provide direct evidence on the properties of the core itself, including shape, while one-neutron transfer to ${ }^{189} \mathrm{~Pb}$ would probe the configuration of intruder states.

Neutron-deficient Se and $\mathrm{Kr}$ nuclei are another classic region of shape coexistence [90,91]. Key nuclei to study are ${ }^{68} \mathrm{Se}$ and ${ }^{70} \mathrm{Se}$. In the former, weak evidence for oblate shape has been found, contrary to what was measured for the latter. The investigation of the excited $0^{+}$states in this region will help to solve this conundrum. In addition, the energies of the first $2^{+}$states show similar behaviour to those in the light $\mathrm{Hg}$ isotopes. To firmly establish the quadrupole moments of the coexisting structures, it is critical to determine the multipole mixing ratios and related $E 0$ components of transitions between states of the same spin. This information can be obtained from angular correlations measured with AGATA combined with conversion-electron measurements.

\subsubsection{Studies of octupole collectivity}

The region of the nuclear chart with the most experimental evidence for octupole deformation is the actinide region, north-east of ${ }^{208} \mathrm{~Pb}$ and centred around ${ }^{224} \mathrm{Ra}$ [92]. This region is also of the greatest interest to searches for a nonzero electric dipole moment (EDM). Two of the candidates for these EDM experiments are ${ }^{221,223} \mathrm{Rn}$, where no excited levels are known. Coulomb excitation of the odd-mass radon and radium isotopes proves to be a much greater challenge than for their even-even neighbours. The high density of lowenergy transitions expected in the $\gamma$-ray spectrum comes from the parity-doublet structure of the rotational bands. AGATA provides excellent angular resolution, which will be crucial when trying to disentangle the complex level schemes of the odd-mass nuclei. The expected large conversion coefficients make heavy odd-mass nuclei ideal objects for studies coupling AGATA and the SPEDE electron spectrometer. With the ability to perform $\mathrm{e}^{-}-\gamma$ coincidences, transition multipolarities can be obtained and used to provide spin and parity assignments, determining the energy of the parity splitting for the first time.

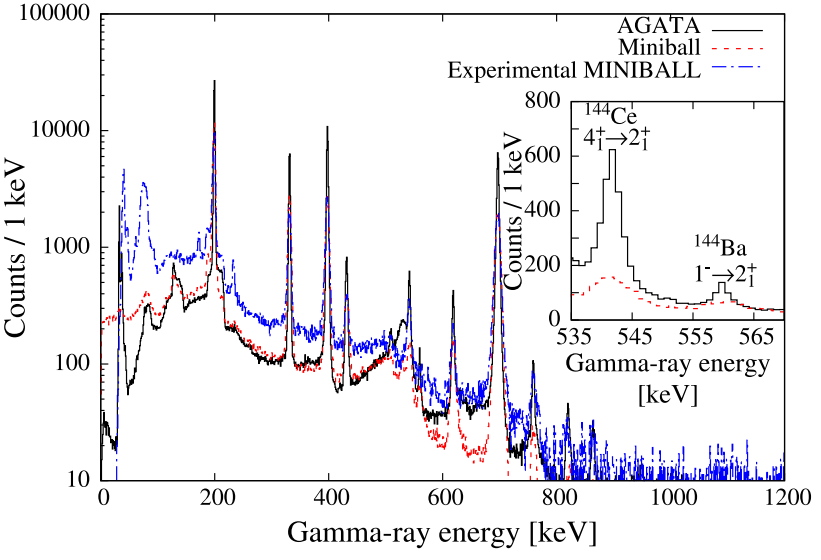

Fig. 15 Comparison of an experimental Miniball spectrum with simulated spectra for AGATA and Miniball of a Coulomb-excitation study of a radioactive ${ }^{144} \mathrm{Ba}$ beam on a ${ }^{208} \mathrm{~Pb}$ target. ${ }^{144} \mathrm{Ba}$ is expected to show strong octupole correlations. The performance gain with AGATA is particularly visible for the weak transitions from the negative parity band (see insert)

In the region of neutron-rich lanthanides, where strong octupole correlations are predicted and were partially confirmed by the level structures, the knowledge of their $E 3$ transition strengths is very limited. Coulomb-excitation measurements have recently been performed on ${ }^{142,144} \mathrm{Ba}$ and ${ }^{142} \mathrm{Xe}$ at HIE-ISOLDE, complementary to the studies of ${ }^{144,146} \mathrm{Ba}$ making use of the CARIBU facility [93,94] at Argonne National Laboratory; it is planned to extend the measurements at HIE-ISOLDE to other octupole-deformed nuclei in this region, for example ${ }^{146,148} \mathrm{Ce}$.

The neutron-rich $\mathrm{Ba}$ beams at both CARIBU and HIEISOLDE suffer from a large amount of isobaric contamination from stable and long-lived isotopes of $\mathrm{Nd}, \mathrm{Ce}$, and $\mathrm{Sm}$. This gives rise to additional $\gamma$-ray transitions and Compton background in the energy spectrum. For example, the $1_{1}^{-} \rightarrow 2_{1}^{+}$transition at $560 \mathrm{keV}$ in ${ }^{144} \mathrm{Ba}$, the intensity of which determines the population of the $1_{1}^{-}$state, lies on the Compton background of the much more intense $2_{1}^{+} \rightarrow 0_{1}^{+}$ transition at $696 \mathrm{keV}$ in ${ }^{144} \mathrm{Nd}$ (see Fig. 15). Fitting the intensity of this weak transition on top of the large background is currently the limiting factor in the uncertainty of the $B(E 3)$ value $(\sim 60 \%)$. Combining AGATA's tracking capabilities with the excellent angular resolution will simultaneously reduce the Compton background and improve the Doppler correction, leading to a reduction in the peak-to-background ratio for such weak transitions. Furthermore, the greater granularity and efficiency will allow $\gamma \gamma$ coincidences to be used for a clean and reliable extraction of the $3_{1}^{-} \rightarrow 2_{1}^{+}$transition intensity in coincidence with the $199-\mathrm{keV}_{1}^{+} \rightarrow 0_{1}^{+}$ transition.

Recently, the first excited $3^{-}$state in ${ }^{132} \mathrm{Sn}$, which lies at an energy of $4352 \mathrm{keV}$, has been populated at HIE-ISOLDE. Another recent Miniball experiment studying Coulomb exci- 
tation of ${ }^{206} \mathrm{Hg}$, an ISOL beam that is unique to the HIEISOLDE facility, shows also tantalising evidence for the $3{ }_{1}^{-}$ state. The much-improved efficiency of the AGATA spectrometer at high energies and for $\gamma \gamma$ coincidences would be again a huge advantage for studying similar states in even more exotic nuclei. In this direction, an observation of the $3_{1}^{-}$states in neutron-rich tin and tellurium isotopes northeast of ${ }^{132} \mathrm{Sn}$ will become feasible with the use of AGATA at HIE-ISOLDE.

\subsection{AGATA at the JYFL facility}

The Accelerator Laboratory at the University of Jyväskylä (JYFL) [16] is one of the leading stable beam facilities in Europe. The K130 cyclotron can deliver a wide range of high-intensity stable beams. Over the last twenty years, the laboratory has supported a world-leading programme of $\gamma$ ray spectroscopy measurements on nuclei at the limits of stability, principally neutron-deficient nuclei. The efficiency of such studies and their ability to address the most exotic nuclei is strongly related to the power of the $\gamma$-ray detector array employed. Installing a near $4 \pi$ AGATA array at JYFL therefore presents the opportunity to drive a paradigm shift in what is possible at JYFL. The full AGATA configuration would be needed for most studies to optimise the efficiency for coincidence $\gamma \gamma$ spectroscopy and the tracking performance.

Several major pieces of apparatus at JYFL can drive an innovative science programme. These include the RITU gasfilled [95] and MARA [96] vacuum-mode recoil separators. The flexibility in having two such systems allows highefficiency studies of heavy nuclei and high-sensitivity studies of exotic lighter nuclei. A further important apparatus is the SAGE system for combined in-beam $\mathrm{e}^{-}-\gamma$ coincidence studies [97], which is unique worldwide.

\subsubsection{Super-heavy elements}

Building on its long-standing track record in decay studies of super-heavy nuclei with the RITU separator, over the last twenty years JYFL has established a dedicated, internationally-leading programme of in-beam studies of super-heavy nuclei in the region around ${ }^{254}$ No. Such studies provide key information on collectivity in this mass region. In addition, locating orbitals close to the Fermi surface provides a discriminating test of nuclear structure models in such super-heavy nuclei and informs the search for a potential island of stability for super-heavy nuclei. The limit so far reached at JYFL was in the study of ${ }^{256} \mathrm{Rf}$ [98] with a production cross section of around $15 \mathrm{nb}$. This study employed the JUROGAM2 [99] germanium array, which has an efficiency of around $5 \%$ at $1.3 \mathrm{MeV}$. The employment of a near- $4 \pi$ AGATA would boost this efficiency to above $30 \%$. With long running periods of a month and 2-3 fold increase in beam current, this would allow heavier nuclei such as ${ }^{260} \mathrm{Sg}$, with a production cross section of a few hundred $\mathrm{pb}$, to be reached for the first time.

Even more importantly, the increase in $\gamma \gamma$ detection efficiency by a factor of 50 with respect to the JUROGAM2 array and the possibility to measure angular correlations and the linear polarisation of photons at all angles, the $\gamma \gamma$ mode will allow detailed spectroscopy of the lighter super-heavy Fm-Rf nuclei (see Fig. 16). In this way known structures can be extended to higher spins, new ones can be observed, and the decay schemes can be established, in particular in odd-mass nuclei whose $\gamma$-ray spectra are notoriously difficult to disentangle. This would provide stringent tests of nuclear structure models in this region and information on the location and evolution of Nilsson orbitals which would have a strong impact on model predictions for super-heavy elements.

\subsubsection{Nuclei along the proton drip line}

There is a strong programme of studies at JYFL along the proton dripline and towards ${ }^{100} \mathrm{Sn}$. In particular, there have been studies of $N=Z-2$ nuclei, which allow isospin-symmetry breaking forces to be studied across $T=1$ isospin triplets. These studies are truly at the limits of what is presently possible with production cross sections for, e.g., ${ }^{70} \mathrm{Kr}$ and ${ }^{74} \mathrm{Sr}$ of around $10 \mathrm{nb}$. To extend these studies to even more proton-rich nuclei and to higher mass, a near-4 $\pi$ AGATA would provide an order of magnitude increased sensitivity. It would also be possible to carry out in-beam studies of nuclei around ${ }^{100} \mathrm{Sn}$, probing the single-particle structure close to this doubly-closed shell nucleus, which is a key test of stateof-the-art nuclear structure models.

The ultimate limit of observable nuclei for odd- $Z$ elements is expected to be determined by direct proton emission. Proton radioactivity is a quantum-tunnelling phenomenon where the barrier penetration probability, and consequently the measured half-life, depends sensitively on the proton-decay $Q$ value, the angular momentum and the size, and shape of the barrier of the emitting state. The approximate spherical symmetry of the heaviest known proton emitters allows a simplified tunnelling description to reproduce partial half-life measurements reasonably well. More sophisticated theoretical approaches have been developed that provide precise predictions for the wave functions of proton-emitting states that account for configuration mixing and residual interactions e.g. [101]. This is particularly important for the halflives of proton-emitting states in highly deformed nuclei where rotational effects, such as Coriolis interactions, change the orbital admixtures that contribute to the nuclear wave function. Hence, a global understanding of proton-unbound nuclei requires a detailed knowledge of the effects of nuclear 

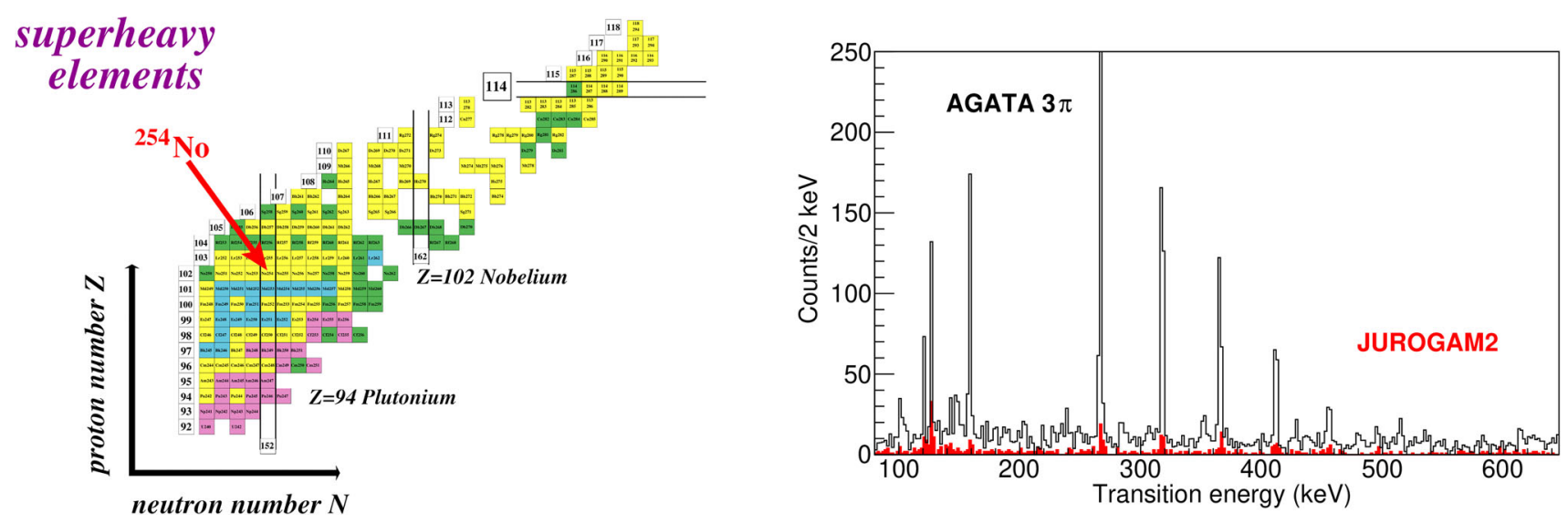

Fig. 16 (Left): Upper end of the nuclear chart showing the location of ${ }^{254} \mathrm{No}$, the current limit for $\gamma \gamma$ spectroscopy. (Right): Comparison of a $\gamma \gamma$ coincidence spectrum of ${ }^{254} \mathrm{No}$ (in coincidence with the $214-\mathrm{keV}$ transition) taken with JUROGAM2 (red) and simulated for a $3 \pi$ AGATA array (black)

structure and dynamics. This requires not only proton emission data, but also information related to the structure of excited states, which can be obtained by measuring their $\gamma$ ray emission using in-beam $p-\gamma$ (or $\alpha-\gamma)$ coincidences (see [102-104]). The high-efficiency of the AGATA spectrometer used in conjunction with the MARA recoil separator could enable measurements of nuclear lifetimes, reduced transition probabilities and quadrupole moments. Such detailed information on the nuclear deformation would provide unprecedented constraints on theoretical models of proton radioactivity.

Consideration of the extreme case of ${ }^{131} \mathrm{Eu}$ shows that lifetime measurements are also feasible for deformed proton-emitting nuclei [105]. The working limit for coincidence recoil-distance Doppler-shift measurements using the angular positions of the JUROGAM detectors suitable for lifetime measurements is $\sim 100 \mu \mathrm{b}$. The production cross section for ${ }^{131} \mathrm{Eu}$ from the ${ }^{58} \mathrm{Ni}\left({ }^{78} \mathrm{Kr}, p 4 n\right)$ is estimated from previous experiments to be $70 \mathrm{nb}$. The $\gamma$-ray detection efficiency of the angles of the AGATA array usable for lifetime measurement is estimated to be $\sim 20 \%$ at $1.3 \mathrm{MeV}$, compared with $1.2 \%$ for the usable angles of JUROGAM. Furthermore, the number of distance settings required could be reduced using a triple-foil plunger. Combining all these factors would allow the required sensitivity limit to study ${ }^{131} \mathrm{Eu}$ to be achieved using AGATA, MARA, and a triple-foil plunger system in a four-week long experiment.

\subsubsection{Studies employing incomplete fusion reactions}

Incomplete fusion reactions, such as multinucleon transfer, deep-inelastic and direct reactions can provide access to regions in the chart of nuclei that so far have not been exploited at RITU or MARA. In very asymmetric reactions, the light fragment is scattered backwards in the centre-of- mass frame, while the heavy partner can be transported to the focal plane of the MARA or RITU separator for identification through recoil-decay tagging. While such studies can provide insight into reaction dynamics, those reactions can also be used to populate excited states in nuclei few nucleons away from the beam- or target-like nuclei. For example, a transfer of six nucleons from beam to target nuclei in the ${ }^{65} \mathrm{Cu}+{ }^{209} \mathrm{Bi}$ reaction has been demonstrated at RITU. This provides access to in-beam spectroscopic studies of nuclei around ${ }^{59} \mathrm{Cr}$ and ${ }^{215} \mathrm{Ra}$. In general, in-beam spectroscopy of neutron-rich nuclei is challenging due to methodological limitations. In particular, apart from manganese nuclei, neutron-rich $23<Z<28$ beams are not available as post-accelerated ISOL beams. The large variety of heavyion beams available at JYFL can be employed to access other regions of light neutron-rich nuclei in a complementary way than ISOL facilities. Examples are ${ }^{44} \mathrm{Ar}$ and the island-ofinversion around ${ }^{32} \mathrm{Mg}$ using $\mathrm{Ca}$ and $\mathrm{S}$ beams, respectively. The near- $4 \pi$ AGATA spectrometer will not only provide much improved $\gamma \gamma$ coincidence efficiency, but its superior position sensitivity will be needed for proper Doppler correction for $\gamma$ rays emitted in flight.

\subsubsection{Study of fission barriers}

Fission is a complex process that involves the competition between the disruptive Coulomb repulsion between the protons and the restoring strong interaction between all nucleons in the nucleus. The way the quantum system evolves towards scission dictates fission time scales and fission-fragment masses and energies.

Fission has been known for many decades now, but to date no theory of fission, based solely on the interactions between protons and neutrons, exists. Yet fission is a fundamental decay process, which plays a crucial role in many domains. 


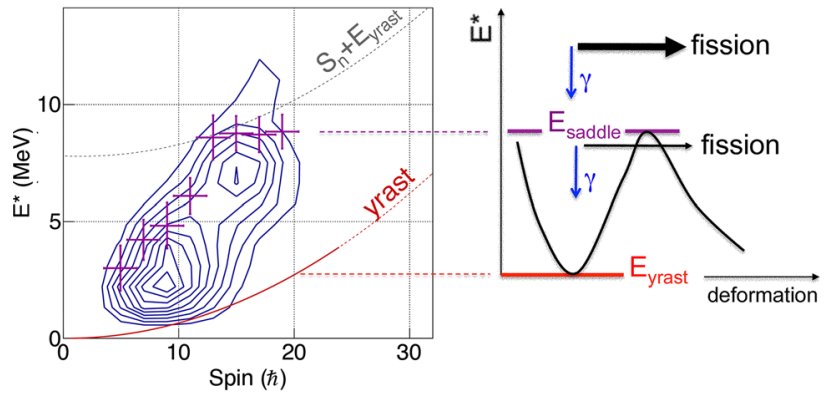

Fig. 17 Entry distribution for the population of ${ }^{254}$ No as function of excitation energy and spin, measured at $219 \mathrm{MeV}$ bombarding energy (left). The solid and dashed lines correspond to the yrast line and neutron separation energy. The crosses, indicating the points where the distribution falls to half of its maximum value, show a saturation above spin 10 and can be directly related to the saddle point energies. The figure on the right shows the potential energy of the nucleus as a function of axial deformation and illustrates how $\gamma$ decay dominates below the saddle point energy while fission takes over at energies above the barrier

It is of course the driving force of the energy production in nuclear reactors. It also governs the stability of transuranium elements and thereby determines the limits of the Segrè chart and the periodic table of elements. Fission prevents the survival of nuclei produced in nuclear reactions and has therefore a strong impact on the experimental synthesis of new heavy isotopes and elements. Finally, fission of very neutronrich very heavy nuclei is a key ingredient for modelling the $r$-process nucleosynthesis $[25,106]$ since it shapes the final $r$-process abundance distribution (see Fig. 9). Whether or not $Z>110$ elements are produced in the $r$-process and can be found in cosmic rays or terrestrial matter $[107,108]$ also depends on the fission properties of these nuclei.

Benchmarking the accuracy and reliability of theoretical models regarding the fission process is therefore required to improve predictions, specially for nuclei away from stability, for which large discrepancies appear between models $[25,109]$. Several observables have been suggested to validate theoretical calculations [110]: fission-fragment mass distributions and kinetic energies, which are sensitive to the shape of the fissioning system, fission isomer excitation energies, fission half-lives, and barrier heights.

Traditionally, fission barrier heights have been measured through the excitation function of fission cross sections induced either by virtual photons, transfer or neutron capture reactions, or following beta (EC) decay. In the region of transfermium nuclei, such techniques cannot be applied due to lack of long-lived targets, beams or unfavourable $Q_{\beta}$ windows. A new method, pioneered at Gammasphere $[55,111]$ can nevertheless be used in the cases where the neutron threshold lies well above the fission barrier. It consists in using the $\gamma$-ray spectrometer as a calorimeter to measure the total energy and spin of the nucleus, which survives fission at various bombarding energies. The saturation of the entry dis- tribution in excitation energy, observed in ${ }^{254} \mathrm{No}$ (see Fig. 17), is due to the onset of fission near the top of the fission barrier and therefore provides a direct measurement of the height as well as the moment of inertia of the saddle point. In this particular case, the extracted fission barrier was found to be $2 \mathrm{MeV}$ lower than the prediction of the Gogny functional [112] and in agreement with microscopic-macroscopic type calculations using the finite range liquid drop model and the folded-Yukawa single-particle model [113].

AGATA in a near- $4 \pi$ configuration coupled to a highacceptance recoil separator is the perfect tool to continue such systematic measurements in super-heavy nuclei produced in fusion-evaporation reactions but also in deep-inelastic reactions. The excellent energy resolution of AGATA in its calorimetric mode will reduce the uncertainty of the extracted barrier heights, which is necessary to constrain models.

\subsection{AGATA at the LNL/SPES facility}

The Laboratori Nazionali di Legnaro (LNL) [17] provides heavy-ion beams from the $15 \mathrm{MV}$ Tandem and from the ALPI superconducting LINAC, the latter either coupled to the Tandem or to the heavy-ion injector PIAVE. Stable beams range from protons to lead with energies up to 10-15 MeV/A.

LNL is currently constructing the ISOL facility, SPES (Selective Production of Exotic Species) [14], dedicated mainly to the production of neutron-rich beams. A proton beam of $40 \mathrm{MeV}$ and $200 \mu \mathrm{A}$ will impinge on an uranium carbide target, producing neutron-rich isotopes as fission fragments, with a rate of $10^{13}$ fissions/s. The neutron-rich products will be extracted in a $1^{+}$charge state, mass separated, boosted to a higher charge-state in ADIGE [114] and accelerated in ALPI up to $10 \mathrm{MeV} / \mathrm{A}$ (see Fig. 18). Intense radioactive beams from the SPES facility will allow a rich $\gamma$-ray spectroscopy program with AGATA, complementary to the experimental investigations with stable beams and to other ISOL facilities.

AGATA at LNL will be able to take advantage of both stable and ISOL beams. A mechanical configuration has been identified which allows using AGATA both with the magnetic spectrometer PRISMA $[115,116]$, and at zero degrees, with ancillary detectors like NEDA [32]. These two configurations already warrant a very rich physics program, which will be further strengthened by the availability of some of the most advanced detectors for light charged particles, e.g., EUCLIDES [27], GRIT [31], SPIDER [117], largevolume scintillators for high-energy $\gamma$ rays, e.g., PARIS [36], conversion-electron spectrometers, and detection systems for selection of evaporation residues, e.g., the Recoil Filter Detector [118].

High-intensity stable beams will allow the exploitation of the extraordinary $\gamma$-ray detection capabilities of AGATA in order to search for rare nuclear phenomena, boosting the 


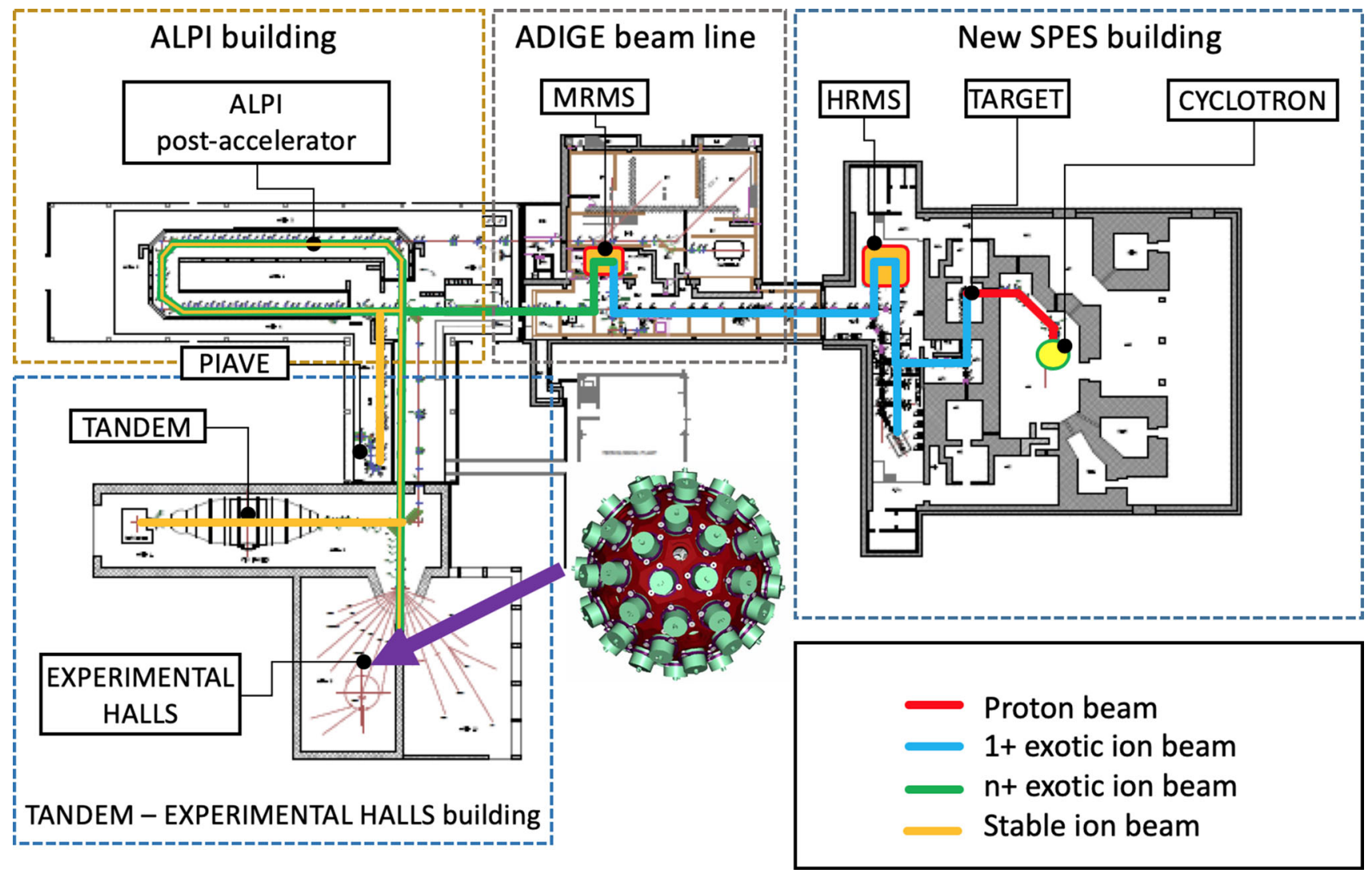

Fig. 18 Layout of the SPES facility at the Laboratori Nazionali di Legnaro and the possible location of AGATA

discovery potential of current research themes carried out at LNL, but also opening new physics programs.

In the following a few highlights of physics cases with AGATA at LNL are presented.

\subsubsection{Collectivity and clusterisation in light nuclei}

The existence of $\alpha$ clusters in light nuclei, from lithium and beryllium, to medium mass nuclei, is a well-known feature, with significant consequences on nucleosynthesis reactions, with the Hoyle state being an eminent case. Nuclear theorists have not yet uncovered the nature of this type of states from first principles [119].

Carrying out investigations of $\gamma$ decays from unbound near-threshold states in light neutron-rich nuclei will provide first information on the onset of collectivity and clusterisation phenomena [120]. This is an almost unexplored territory and AGATA will allow to access weak electromagnetic decays from such states in neutron-rich $\mathrm{B}, \mathrm{C}$, $\mathrm{N}$, and $\mathrm{O}$ nuclei, with decay branches of the order of $10^{-3}-10^{-5}$. Fusion reactions induced by intense Li beams on $\mathrm{Li}, \mathrm{Be}, \mathrm{B}$, and $\mathrm{C}$ targets, followed by the evaporation of a single charged particle, detected in GRIT, will be exploited.
Light neutron-rich nuclei are also ideal to test the details of the nuclear force, in particular the three-body term of the nucleon-nucleon interaction. Here, the focus is on the decay properties of bound excited states in exotic systems like ${ }^{20,22} \mathrm{O}$ and ${ }^{16,18} \mathrm{C}$, which can be reached in deep-inelastic reactions using intense ${ }^{18} \mathrm{O}$ beam on heavy targets. These kinds of studies will benefit from the large angular coverage of AGATA coupled to the PRISMA magnetic spectrometer, significantly improving the sensitivity obtained in early AGATA experiments [121] (see Fig. 19).

\subsubsection{Isospin symmetry breaking in $N \sim Z$ nuclei}

Isospin symmetry breaking can be addressed via measurements of energies of excited states and electromagnetic decay properties in $N=Z$ and in mirror nuclei. Lifetime measurements will allow (i) to access isospin forbidden $(\Delta T=0)$ $E 1$ transitions in heavy $N=Z$ nuclei, following the study of ${ }^{64} \mathrm{Ge}$ [57], (ii) to study proton-neutron isoscalar $T=0$, $S=1$ correlations in $N=Z$ nuclei populated up to high angular momenta, (iii) to measure electromagnetic decay properties of yrast states in $N=Z$ systems like ${ }^{88} \mathrm{Ru},{ }^{90} \mathrm{Rh}$, ${ }^{92} \mathrm{Pd}$ and finally (iv) the GDR will be used to probe the dependence of the isospin mixing on temperature (e.g., in 


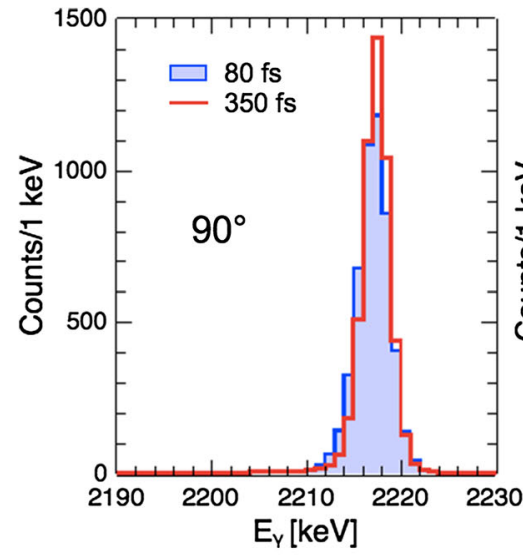

Fig. 19 Simulated lineshapes of the 2217-keV transition deexciting the second $2^{+}$state of the hard-to-reach nucleus ${ }^{16} \mathrm{C}$, produced in the deep-inelastic reaction ${ }^{18} \mathrm{O}+{ }^{181} \mathrm{Ta}$ at $\mathrm{v} / \mathrm{c} \sim 10 \%$. Lifetime values of 80 $\mathrm{fs}$ and $350 \mathrm{fs}$ are considered, as predicted by ab initio theories, including
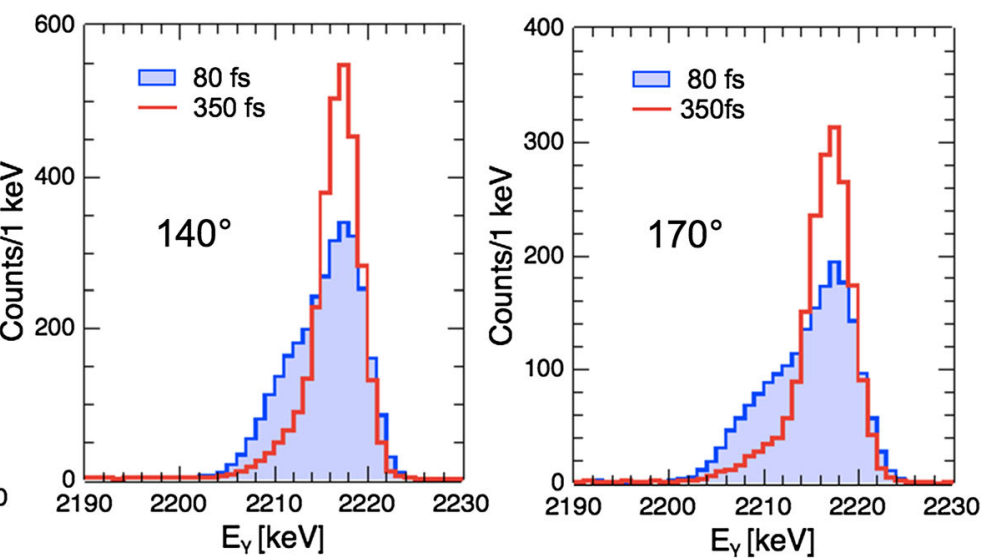

two-body or two+three-body interactions in the nuclear force, respectively. While the AGATA measurement at $90^{\circ}$ (left panel) would provide the most precise value of the $\gamma$ transition, sensitivity to lifetimes will be reached at backward angles (see panels for $140^{\circ}$ and $170^{\circ}$ )
${ }^{52} \mathrm{Fe}$ ), following previous studies with AGATA at LNL on ${ }^{80} \mathrm{Zr}$ [122]. Fusion-evaporation reactions will be employed, in synergy with the radioactive beam program. Very intense light radioactive beams of SPES, e.g., ${ }^{7} \mathrm{Be}$, give access to $N=Z$ nuclei produced in fusion-evaporation reactions with cross-sections down to about $10 \mu \mathrm{b}$.

One of the most exciting cases is the $T=3 / 2$ isospin quadruplet ${ }_{24}^{45} \mathrm{Cr}_{21}-{ }_{23}^{45} \mathrm{~V}_{22}-{ }_{22}^{45} \mathrm{Ti}_{23}-{ }_{21}^{45} \mathrm{Sc}_{24}$ and, in particular, its most exotic member, the $T_{z}=-3 / 2$ nucleus ${ }^{45} \mathrm{Cr}$, with the largest $Z$ difference between the mirror nuclei. Studies of its excited states would give information on Coulomb energy differences and isospin non-conserving phenomena, which can be compared with large-scale shell-model calculations. AGATA coupled to the neutron detector array NEDA and a $4 \pi$ charged-particle array will push these studies far beyond their current limits.

\subsubsection{Correlations in valence nucleons and the emergence of complex excitations}

Nuclei, which are a few particles and/or holes away from the shell closures at $N=82$ and $Z=50$, are of particular interest to investigate shell-structure changes in very neutronrich nuclei. In this context, studies of the one-valence proton ${ }^{134,135} \mathrm{Sb}$ isotopes with one proton and one or two neutrons outside the doubly-magic ${ }^{132} \mathrm{Sn}$ nucleus, may provide one of most sensitive tests of theoretical calculations, i.e., the direct comparison of transition matrix elements measured with low-energy Coulomb excitation using intense SPES beams (and complementary to experiments at relativistic energies at FAIR) with their predicted values depending on the matrix elements of the proton-neutron interaction between different major shells. Cluster-transfer reactions with $\mathrm{Sn}$ beams on weakly-bound ${ }^{7} \mathrm{Li}$ targets [123] will further extend these studies to higher excitation energies (see Fig. 3 in Sect. 2.1).

Systems built of one or two valence particle(s) or hole(s) and a doubly magic core are also ideal to study the coupling between single-particle excitations and collective responses of the nucleus and phonons in particular. Such couplings are considered the major source of partial occupancies of nucleonic orbitals and the doorway to damping phenomena in giant resonances [124]. Systems like ${ }^{130} \mathrm{Cd},{ }^{130-132} \mathrm{In}$, ${ }^{130-134} \mathrm{Sn}$, and ${ }^{132-134} \mathrm{Sb}$, around the doubly-magic ${ }^{132} \mathrm{Sn}$ core, are the most suited for the SPES facility, where pure and intense Sn-like beams will be available. A multifaceted scenario is expected to appear with increasing spin, pointing to a hybrid nature of these excitations [125], in analogy to similar phenomena known in other branches of physics, including condensed matter [126]. The coupling of AGATA with the PRISMA spectrometer and, possibly, with light chargedparticle detection devices will allow very selective studies using transfer and cluster-transfer reactions. Fission reactions induced by a stable heavy beam, such as ${ }^{208} \mathrm{~Pb}$, impinging on a light target could offer, in addition, the possibility to populate nuclei in the ${ }^{78} \mathrm{Ni}$ region $(N=50)$, reaching states with energy and angular momentum high enough to observe $N=50$ core-breaking states and/or single particle states in the large- $j$ shells above $N=50$.

\subsubsection{Shape coexistence and shape evolution}

Exotic nuclei produced at SPES will offer the opportunity to investigate in great detail the phenomenon of shape coexistence and its extreme manifestation as shape isomerism [127]. Retarded $\gamma$-decay branches between different configurations are fingerprints of this phenomenon, which provide a 


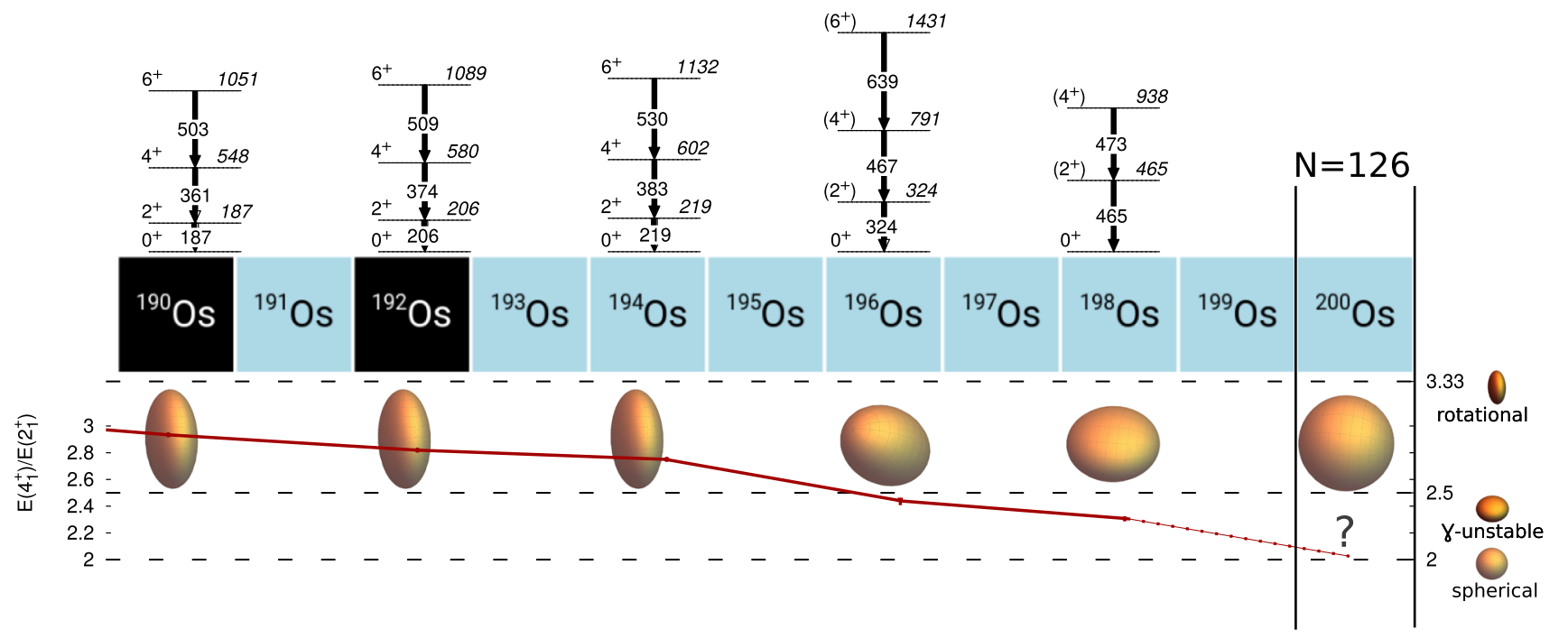

Fig. 20 Yrast band excitation energies for the isotopes ${ }^{190-198}$ Os. The experimental ratio of the energies of the $4_{1}^{+}$and $2_{1}^{+}$states indicates a shape evolution from a prolate $\left({ }^{190} \mathrm{Os}\right)$ to an oblate shape $\left({ }^{198} \mathrm{Os}\right)$, passing by an almost perfect gamma-unstable/triaxial rotor ( $\left.{ }^{196} \mathrm{Os}\right)$. The red

powerful insight into the microscopic origin of nuclear deformation, when experimental data are compared with sophisticated (beyond) mean-field, large-scale shell-model [128], and most recently Monte-Carlo Shell-Model calculations [129]. The most promising cases, predicted by various theoretical approaches [130-132] are the Cd and Pd nuclei with $A=112-118$, which can be studied at SPES by one-proton transfer reactions, using intense $\mathrm{Ag}$ beams with $A=113$ 119 on light or heavier targets, e.g., ${ }^{10,11} \mathrm{~B}$ or ${ }^{93} \mathrm{Nb}$, with GRIT and/or PRISMA to select the reaction products.

The evolution of the nuclear shape will also be studied in neutron-rich nuclei near $N=60$ and with $Z=36-40$ where the most dramatic shape change in the nuclear chart has been observed (see Sect. 2.3), showing the typical characteristics of a quantum-phase transition [133], with strong impact on the $r$-process nucleosynthesis path as well. Neutron-rich $\mathrm{Kr}$ and $\mathrm{Rb}$ nuclei, produced at SPES with very high intensities, will enable extremely detailed and precise Coulombexcitation studies to measure quadrupole moments and to search for signatures of configuration inversion and shape coexistence near $N=60$. Experimental information on configuration mixing and triaxiality in ${ }^{90-98} \mathrm{Kr}$ and ${ }^{78-82} \mathrm{Ge}$ nuclei will provide important benchmarks for beyond-meanfield and large-scale shell-model calculations. The unprecedented Doppler-correction capabilities of AGATA in combination with the highly segmented SPIDER Si detector array will be particularly beneficial for Coulomb-excitation studies of odd-mass nuclei, when many $\gamma$-ray transitions are expected in a narrow energy range.

Intense ${ }^{94-96} \mathrm{Sr}$ and ${ }^{94-96} \mathrm{Rb}$ beams from SPES used to induce cluster-transfer reactions on a ${ }^{7} \mathrm{Li}$ target will be the solid line indicates the experimental $\mathrm{E}\left(4_{1}^{+}\right) / \mathrm{E}\left(2_{1}^{+}\right)$ratio and the dashed lines the limits for an axial rotor, a vibrational nucleus and a gammaunstable/triaxial rotor

ideal tool to access otherwise hard-to-reach excited structures built on isomeric states and associated with different intrinsic configurations, in particular in the case of the most complex odd-odd systems (see Fig. 4). By detecting the $\alpha$ particle emitted in the reaction process in GRIT and the final reaction products (e.g., ${ }^{96,97} \mathrm{Y}$ and ${ }^{96,97} \mathrm{Sr}$ ) in PRISMA an unprecedented level of sensitivity could be reached. Finally, shape evolution including a passage through triaxial shapes has also been observed in heavier mass regions [134] and could be studied in further detail by multinucleon transfer reactions (see Fig. 20) in combination with lifetime measurements $[135,136]$.

The appearance of very elongated, superdeformed (SD) shapes, in rapidly rotating, highly excited nuclei is one of the most striking examples of shape changes within the same nucleus. The existence of even more elongated shapes, named hyper-deformed (HD), still needs to be experimentally confirmed. Other extreme phenomena occurring at high excitation energies are Jacobi- and Poincare-shape transitions; they can be tackled by investigating the GDR strength function [22]. Up to now, the limiting factors were the strong fission competition and limited detection efficiency. In experiments with neutron-rich beams at SPES the fission competition is expected to be largely reduced. Using high intensity neutronrich $\mathrm{Sn}$-like beams and a ${ }^{48} \mathrm{Ca}$ target compound nuclei will be populated well above spin $80 \hbar$, leading to residua with spins around $70 \hbar$, where HD and SD states are expected to be populated. Reaching such ultra-high spins will also enable searches for shape transitions towards fission, via a series of elongated triaxial shapes, as reported in the ${ }^{160} \mathrm{Er}$ region $[137,138]$. AGATA coupled to scintillator arrays, such as 
PARIS, for improved high-energy detection efficiency, will provide the required detection efficiency and sensitivity.

\subsubsection{Pygmy resonances}

Pygmy dipole resonances (PDR) are associated with oscillations of a neutron skin against the core. They will be studied at LNL in an exotic region of the nuclear chart, following their evolution along isotopic chains. Pygmy states in stable systems have been investigated at LNL using inelastic scattering with ${ }^{17} \mathrm{O}$ heavy ions (at $20 \mathrm{MeV} / \mathrm{A}$ ) [139] and the very first implementation of AGATA, coupled with segmented silicon detectors of the TRACE project [29]. A new mode of nuclear excitation, the Pygmy Quadrupole Resonance (PQR), associated with a concentration of low-energy electric quadrupole strength, was also observed [140]. Similar experiments could be performed with exotic beams in inverse kinematics at the highest available incident energies (about $10 \mathrm{MeV} / \mathrm{A}$ ), where appreciable cross sections are still expected. Here, $\gamma$-ray tracking capabilities will be fundamental to provide an optimal Doppler correction.

Appropriate cases will be neutron-rich ${ }^{90,92,94} \mathrm{Sr}$ isotopes, produced at SPES with high intensities, which could be probed by inelastic scattering reactions, e.g., $\left(\alpha, \alpha^{\prime} \gamma\right)$ and $\left(p, p^{\prime} \gamma\right)$, on solid or liquid targets, using AGATA coupled to highly granular particle detectors, such as GRIT [31]. Stable systems, such as ${ }^{88} \mathrm{Sr}$, would be an important point of reference, to be investigated in detail by stable beam and target combinations (see Fig. 8). By performing $(d, p)$ reactions with intense ${ }^{132} \mathrm{Sn}$-like beams, one might also access PDR states by transfer reactions, obtaining information on the related single-particle strength. Finally, SPES beams could provide the opportunity to search for the predicted separation of the PDR peak in deformed nuclei into two bumps [141], as it occurs for the GDR peak.

\subsubsection{Proton-rich nuclei}

The most intense light radioactive beams from SPES, apart from ${ }^{10} \mathrm{Be}$, lie on the proton-rich side, offering the chance to study new phenomena that may arise from a large proton excess. The keywords are proton-proton correlations, halo effects, pairing, two-proton emission, at or close to the proton drip line. Very interesting cases are ${ }^{9} \mathrm{C}$ and ${ }^{28} \mathrm{~S}$, populated by reactions like ${ }^{7} \mathrm{Be}\left({ }^{3} \mathrm{He}, n\right){ }^{9} \mathrm{C}$ and ${ }^{26} \mathrm{Si}\left({ }^{3} \mathrm{He}, n\right){ }^{28} \mathrm{~S}$, which would require the use of a powerful neutron detector. While ${ }^{9} \mathrm{C}$ is a candidate for being a halo nucleus, i.e., having one proton more than the suspected one-proton halo nucleus ${ }^{8} \mathrm{~B}$ [142], strong correlations have been found between the two protons emitted from high-lying states in ${ }^{28} \mathrm{~S}$ [143]. Detailed spectroscopic studies of the configuration of these states will shed light on the role of pairing close to the proton dripline.

\subsubsection{Nuclear astrophysics}

Astrophysical research at SPES will mainly focus on the study of neutron-capture reactions, essential for a quantitative understanding of the element formation in the universe. In the region around doubly-magic ${ }^{132} \mathrm{Sn}$, astrophysically relevant neutron-capture cross sections for the $r$-process could be measured by using indirect techniques, like the surrogate method [81]. Early experiments at SPES could be $(d, p)$ or $(d, t)$ transfer reactions on ${ }^{129,131,133,134} \mathrm{Sn}$ and ${ }^{78,80,81} \mathrm{Ga}$.

Relevant physics cases for the $s$-process are, for example, the neutron captures on the ${ }^{79} \mathrm{Se}$ and ${ }^{85} \mathrm{Kr}$ branching nuclei, whose cross sections together with the observed element abundances allow to estimate the neutron density at the $s$-process site: depending on the neutron density, different $s$-process patterns are expected. On the proton-rich side, astrophysically important nuclei involved in the $r p$-process nucleosynthesis could be also explored with the available ${ }^{25,26} \mathrm{Al}$ beams, using indirect approaches such as $\left({ }^{3} \mathrm{He}, d\right)$ or $(d, n)$ reactions. Knowledge of resonant states in ${ }^{26} \mathrm{Si}$ and their $\gamma$-decay branches are key to constrain the reaction rate of the ${ }^{25} \mathrm{Al}(p, \gamma)^{26} \mathrm{Si}$ reaction that is required to understand the synthesis of ${ }^{26} \mathrm{Al}$ in explosive hydrogen burning. Indirect $(d, p)$ reactions can also be used to obtain information on single-particle resonances in their mirror partners. The use of AGATA combined with a thick cryogenic target will constitute a breakthrough in this type of studies.

\section{Conclusions}

The exciting science programme to be performed with AGATA is described in this paper. AGATA will have an enormous impact on nuclear structure studies at the extremes of isospin, mass, angular momentum, excitation energy and temperature. It will enable us to uncover hitherto hidden secrets of the atomic nucleus.

Its importance was also recognised in several long-range plans for nuclear physics, including the 2017 NuPECC Long Range Plan where the completion of AGATA array is one of the highlight recommendations. AGATA is a continually evolving international detector facility. The collaboration is aiming for completion of the full $4 \pi$ array, consisting of 180 detectors by $\sim 2030$. AGATA is expected to be hosted by all major European nuclear-structure facilities in the coming decade and beyond. The AGATA collaboration plans, through careful liaison with the management of the facilities, to ensure it will be located at each facility at the optimum time to maximise their science output. This dynamic 
approach within the AGATA collaboration will allow its scientists to exploit the very best opportunities.

Acknowledgements The AGATA project has been supported by the European Structural Funds FSE FEDER, the Academy of Finland FIRI program, Finland, the Direction de Recherches Fondamentales of CEA, France, the Institut National de Physique Nucléaire et de Physique des Particules (IN2P3) of CNRS, France, the Bundesministerium für Bildung und Forschung (BMBF), Germany, the Institute of Nuclear Research of the Hungarian Academy of Sciences (ATOMKI), Hungary, the Istituto Nazionale di Fisica Nucleare (INFN), Italy, the National Science Center ( $\mathrm{NCN}$ ), Poland, the Knut and Alice Wallenberg foundation (Sweden), the Swedish Research Council, the Conselleria d'Educació, Investigació, Cultura i Esport, Generalitat Valenciana and Ministerio de Ciencia, Innovación y Universidades, Spain, the Türkiye Bilimsel ve Teknolojik Araştirma Kurumu (TÜBITAK), Turkey, and the United Kingdom Research and Innovation - Science and Technology Facilities Council (UKRI-STFC).

Data Availability Statement This manuscript has no associated data or the data will not be deposited. [Authors' comment: Data sharing not applicable to this article as no datasets were generated or analysed during the current study.]

Open Access This article is licensed under a Creative Commons Attribution 4.0 International License, which permits use, sharing, adaptation, distribution and reproduction in any medium or format, as long as you give appropriate credit to the original author(s) and the source, provide a link to the Creative Commons licence, and indicate if changes were made. The images or other third party material in this article are included in the article's Creative Commons licence, unless indicated otherwise in a credit line to the material. If material is not included in the article's Creative Commons licence and your intended use is not permitted by statutory regulation or exceeds the permitted use, you will need to obtain permission directly from the copyright holder. To view a copy of this licence, visit http://creativecomm ons.org/licenses/by/4.0/.

\section{References}

1. F. Beck et al., Prog. Part. Nucl. Phys. 28, 443 (1992)

2. J. Simpson et al., Z. Phys. A358, 139 (1997)

3. M. A. Delaplanque, R. M. Diamond (Eds.), Gammasphere Proposal, Preprint LBNL-5202 (1987)

4. J. Eberth et al., Prog. Part. Nucl. Phys. 46, 389 (2001)

5. N. Warr et al., Eur. Phys. J. A49, 40 (2013)

6. J. Simpson et al., Acta Phys. Hung. N. S. 11, 159 (2000)

7. A. Akkoyun et al., Nucl. Instrum. Methods Phys. Res. A668, 26 (2012)

8. I.Y. Lee, Nucl. Instrum. Methods Phys. Res. A422, 195 (1999)

9. GRETA (Gamma-Ray Energy Tracking Array) Conceptual Design Report. http://greta.lbl.gov/documents (2017)

10. A. Korichi, T. Lauritsen, Eur. Phys. J. A55, 121 (2019)

11. https://fair-center.eu/

12. https://fair-center.eu/for-users/experiments/nustar/super-frs. html

13. http://hie-isolde-project.web.cern.ch

14. F. Gramegna, Il Nuovo Cimento 42C, 61 (2019). https://web.infn. $\mathrm{it} /$ spes/

15. https://www.ganil-spiral2.eu/en/

16. https://www.jyu.fi/science/en/physics/research/infrastructures/ accelerator-laboratory
17. G. de Angelis, G. Fiorentini, Phys. Scr. 91, 113001 (2016). https:// www.lnl.infn.it

18. NUPECC Long Range Plan (2017). http://www.nupecc.org

19. L. Coraggio et al., Prog. Part. Nucl. Phys. 62, 135 (2009)

20. B. Blank et al., Nucl. Instrum. Methods Phys. Res. B416, 41 (2018)

21. B. Herskind et al., Acta Phys. Polon. B38, 1421 (2007)

22. A. Maj et al., Nucl. Phys. A731, 319 (2004)

23. L. Pellegri et al., Phys. Lett. B738, 51 (2014)

24. O. Wieland et al., Phys. Rev. Lett. 102, 092502 (2009)

25. S. Goriely, G. Martínez-Pinedo, Nucl. Phys. A944, 158 (2015)

26. J. Scheurer et al., Nucl. Instrum. Methods Phys. Res. A385, 501 (1997)

27. D. Testov et al., Eur. Phys. J. A55, 87 (2019)

28. Ch. Theisen et al., Nucl. Instrum. Methods Phys. Res. A747, 69 (2014)

29. D. Mengoni et al., Nucl. Instrum. Methods Phys. Res. A764, 241 (2014)

30. E.C Pollacco et al., Eur. Phys. J. A25, 287 (2005). http://must2. cea.fr

31. http://grit.in2p3.fr/

32. T. Huyuk et al., Eur. Phys. J. A52, 55 (2016)

33. Ö. Skeppstedt et al., Nucl. Instrum. Methods Phys. Res. A421, 531 (1999)

34. J. Ljungvall et al., Nucl. Instrum. Methods Phys. Res. A528, 741 (2004)

35. O.J. Roberts et al., Nucl. Instrum. Methods Phys. Res. A748, 91 (2014)

36. A. Maj et al., Acta Phys. Polon. B40, 565 (2009)

37. https://fair-center.eu/user/experiments/nustar/experiments/ hispecdespec

38. P. Golubev et al., Nucl. Instrum. Methods Phys. Res. A723, 55 (2013)

39. DEGAS Technical Design Report. https://fair-center.eu/forusers/experiments/nustar/documents/technical-design-reports. html

40. D. Brink, Ph.D. thesis, Oxford University (1955) (unpublished)

41. P. Axel, Phys. Rev. 126, 671 (1962)

42. D. Martin et al., Phys. Rev. Lett. 119, 182503 (2017)

43. P.J. Davies et al., Phys. Rev. Lett. 111, 072501 (2013)

44. C.B. Hinke et al., Nature 486, 341 (2012)

45. D. Bazin et al., Phys. Rev. Lett. 101, 252501 (2008)

46. M. Górska et al., Phys. Rev. Lett. 79, 2415 (1997)

47. S. Pullanhiotan et al., Nucl. Instrum. Methods Phys. Res. A593, 343 (2008)

48. M. Rejmund et al., Nucl. Instrum. Methods Phys. Res. A646, 184 (2011)

49. J.J. Valiente-Dobón et al., Nucl. Instrum. Methods Phys. Res. A927, 81 (2019)

50. J.-N. Scheurer et al., Nucl. Instrum. Methods Phys. Res. A385, 501 (1997)

51. Ch. Schmitt et al., Nucl. Instrum. Methods Phys. Res. A621, 558 (2010)

52. Ch. Theisen, EPJ Web Conf. 163, 00059 (2017)

53. R. Anne et al., Nucl. Instrum. Methods Phys. Res. A257, 215 (1987)

54. D. Ackermann, C. Theisen, Phys. Scr. 92, 083002 (2017)

55. G. Henning et al., Phys. Rev. Lett. 113, 262505 (2014)

56. S.M. Lenzi et al., Phys. Rev. C98, 054322 (2018)

57. E. Farnea et al., Phys. Lett. B551, 56 (2003)

58. B. Cederwall et al., Nature 469, 68 (2011)

59. D.D. Warner, M.A. Bentley, P. Van Isacker, Nat. Phys. 2, 311 (2006)

60. P. Van Isacker, D.D. Warner, A. Frank, Phys. Rev. Lett. 94, 162502 (2005)

61. D. Rudolph et al., Phys. Rev. C63, 021301 (2000) 
62. A. Johnson, H. Ryde, J. Sztarkier, Phys. Lett. B34, 605 (1971)

63. R.H. Cyburt et al., Astrophys. J. 830, 55 (2016)

64. H. Abusara, A.V. Afanasjev, Phys. Rev. C79, 024317 (2009)

65. A. Maj et al., Nucl. Phys. A731, 319 (2004)

66. N. Schunck, J. Dudek, B. Herskind, Phys. Rev. C75, 054304 (2007)

67. M.J.A. de Voigt, J. Dudek, Z. Szymański, Rev. Mod. Phys. 55, 949 (1983)

68. http://isolde.web.cern.ch

69. W. Rother et al., Phys. Rev. Lett. 106, 022502 (2011)

70. K.T. Flanagan et al., Phys. Rev. Lett. 103, 142501 (2009)

71. P. Morfouace et al., Phys. Lett. B751, 306 (2015)

72. X.F. Yang et al., Phys. Rev. Lett. 116, 182502 (2016)

73. A. Banu et al., Phys. Rev. C72, 061306 (2005)

74. J. Cederkäll et al., Phys. Rev. Lett. 98, 172501 (2007)

75. C. Vaman et al., Phys. Rev. Lett. 99, 162501 (2007)

76. A. Ekström et al., Phys. Rev. Lett. 101, 012502 (2008)

77. L. Corragio et al., Phys. Rev. C91, 041301(R) (2015)

78. T. Togashi et al., Phys. Rev. Lett. 121, 062501 (2018)

79. D.C. Radford et al., Phys. Rev. Lett. 88, 222501 (2002)

80. D. Kocheva et al., Phys. Rev. C96, 044305 (2017)

81. J.E. Escher, J.T. Burke, F.S. Dietrich et al., Rev. Mod. Phys. 84, 353 (2012)

82. R. Hatarik et al., Phys. Rev. C81, 011602(R) (2010)

83. R. Tribble et al., Rep. Prog. Phys. 77, 106901 (2014)

84. A. Tumino et al., Nature 557, 687 (2018)

85. C. Spitaleri et al., Eur. Phys. J. A55, 161 (2019)

86. S. Cherubini et al., Phys. Rev. C92, 015805 (2015)

87. L. Lamia et al., Astrophys. J. 879, 23 (2019)

88. P. Papadakis et al., Eur. Phys. J. A54, 42 (2018)

89. A.N. Andreyev et al., Nature 405, 430 (2000)

90. E. Bouchez et al., Phys. Rev. Lett. 90, 082502 (2003)

91. E. Clément et al., Phys. Rev. C75, 054313 (2007)

92. L.P. Gaffney et al., Nature 497, 199 (2013)

93. B. Bucher et al., Phys. Rev. Lett. 116, 112503 (2016)

94. B. Bucher et al., Phys. Rev. Lett. 118, 152504 (2017)

95. M. Leino et al., Nucl. Instrum. Methods Phys. Res. B99, 653 (1995)

96. https://www.jyu.fi/science/en/physics/research/infrastructures/ accelerator-laboratory/nuclear-physics-facilities/ recoil-separators/mara-mass-analysing-recoil-apparatus

97. J. Pakarinen et al., Eur. Phys. J A50, 53 (2014)

98. P.T. Greenlees et al., Phys. Rev. Lett. 109, 012501 (2012)

99. https://www.jyu.fi/science/en/physics/research/infrastructures/ accelerator-laboratory/nuclear-physics-facilities/copy_of_ nuclear-spectroscopy

100. F.D. Becchetti, G.W. Greenlees, Phys. Rev. 182, 1190 (1969)

101. A.T. Kruppa et al., Phys. Rev. Lett. 84, 4549 (2000)

102. D. Rudolph et al., Phys. Rev. Lett. 80, 3018 (1998)

103. D. Rudolph et al., Phys. Rev. Lett. 82, 3763 (1999)

104. D. Rudolph et al., Phys. Rev. Lett. 86, 1450 (2001)

105. D. Rudolph et al., Phys. Rev. C63, 021301(R) (2001)

106. I. Petermann et al., Eur. Phys. J. A48, 122 (2012)

107. G.M. Ter-Akopian, S.N. Dimitriev, Nucl. Phys. A944, 177 (2015)

108. G. Korschinek, W. Kutschera, Nucl. Phys. A944, 190 (2015)

109. A. Mamdouh et al., Nucl. Phys. A679, 337 (2001)

110. G.F. Bertsch et al., J. Phys. G Nucl. Part. Phys. 42, 077001 (2015)

111. P. Reiter et al., Phys. Rev. Lett. 84, 3542 (2000)

112. J.L. Egido, L.M. Robledo, Phys. Rev. Lett. 85, 1198 (2000)

113. P. Möller et al., Phys. Rev. C79, 064304 (2009)

114. A. Galat et al., Proc. 23rd Int. workshop on ECR ion sources. J. Instrum. 13, C12009 (2018)

115. A.M. Stefanini et al., Nucl. Phys. A701, 217c (2002)

116. A.D. Montanari et al., Eur. Phys. J. A47, 4 (2011)

117. M. Rocchini et al., Phys. Scr. 92, 074001 (2017)
118. W. Meczyński et al., Nucl. Instrum. Methods Phys. Res. A580, 1310 (2007)

119. E. Epelbaum, H. Krebs, D. Lee, Ulf-G Meissner, Phys. Rev. Lett. 106, 192501 (2011)

120. J. Okołowicz, M. Płoszajczak, W. Nazarewicz, Prog. Theor. Phys. Suppl. 196, 230 (2012)

121. M. Ciemała et al., Phys. Rev. C (in press)

122. S. Ceruti et al., Phys. Rev. Lett. 115, 222502 (2015)

123. S. Bottoni et al., Phys. Rev. C92, 024322 (2015)

124. P.F. Bortignon, A. Bracco, R.A. Broglia, Giant Resonances: Nuclear Structure at Finite Temperature (Harwood Academic, New York, 1998)

125. G. Bocchi et al., Phys. Lett. B760, 273 (2016)

126. R.A. Broglia, G. Colò, G. Onida, H.E. Roman, Solid State Physics of Finite Systems (Springer, Berlin, 2004)

127. S. Leoni et al., Phys. Rev. Lett. 118, 162502 (2017)

128. E. Caurier et al., Rev. Mod. Phys. 77, 427 (2005)

129. Y. Tsunoda et al., Phys. Rev. C89, 031301(R) (2014)

130. P. Möller et al., Phys. Rev. Lett. 103, 212501 (2009)

131. B. Nerlo-Pomorska et al., Eur. Phys. J. A53, 67 (2017)

132. S. Quan, Q. Chen, Z.P. Li, T. Nikšić, D. Vretenar, Phys. Rev. C95, 054321 (2017)

133. T. Togashi et al., Phys. Rev. Lett. 117, 172502 (2016)

134. P.R. John et al., Phys. Rev. C95, 064321 (2017)

135. A. Dewald, O. Möller, P. Petkov, Prog. Part. Nucl. Phys. 67, 186 (2012)

136. J. Valiente Dobón et al., Phys. Rev. Lett. 102, 242502 (2009)

137. E.S. Paul et al., Phys. Rev. Lett. 98, 012501 (2007)

138. A.V. Afanasjev et al., Phys. Rev. C86, 031304(R) (2012)

139. A. Bracco, F.C.L. Crespi, E.G. Lanza, Eur. Phys. J. A51, 99 (2015)

140. L. Pellegri et al., Phys. Rev. C92, 014330 (2015)

141. D. Peña Arteaga, E. Khan, P. Ring, Phys. Rev. C79, 034311 (2009)

142. R.E. Warner et al., Phys. Rev. C52, 1166(R) (1995)

143. X.X. Xu et al., Phys. Lett. B727, 126 (2013)

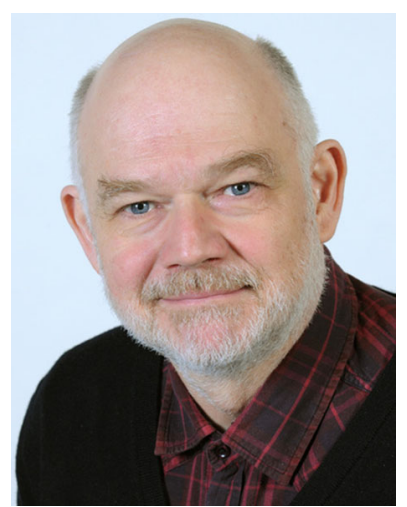

W. Korten is an experimental physicist working in the field of nuclear structure physics. He received his doctoral degree in 1988 from the University of Heidelberg. After post-doctoral positions in Berkeley and Copenhagen he received his habilitation from the University of Bonn on studies of superdeformed nuclei with the EUROBALL spectrometer. Since 1996, he has been researcher at CEA ParisSaclay, France, and was nominated Directeur de Recherche in

2012. He has been involved since the beginning in the AGATA project and chaired the editorial board for this review article, which is based on contributions from members of the AGATA collaboration. 